\title{
Genetic susceptibility to bone and soft tissue sarcomas: a field synopsis and meta-analysis
}

\author{
Clara Benna ${ }^{1,2}$, Andrea Simioni ${ }^{1}$, Sandro Pasquali ${ }^{1,4}$, Davide De Boni ${ }^{1}$, Senthilkumar \\ Rajendran', Giovanna Spiro', Chiara Colombo4, Calogero Virgone ${ }^{5}$, Steven G. \\ DuBois ${ }^{6}$, Alessandro Gronchi ${ }^{4}$, Carlo Riccardo Rossi ${ }^{1,3}$ and Simone Mocellin ${ }^{1,3}$ \\ ${ }^{1}$ Department of Surgery Oncology and Gastroenterology, University of Padova, Padova, Italy \\ ${ }^{2}$ Clinica Chirurgica I, Azienda Ospedaliera Padova, Padova, Italy \\ ${ }^{3}$ Surgical Oncology Unit, Istituto Oncologico Veneto (IOV-IRCCS), Padova, Italy \\ ${ }^{4}$ Sarcoma Service, Department of Surgery, Fondazione IRCCS Istituto Nazionale dei Tumori, Milano, Italy \\ ${ }^{5}$ Pediatric Surgery, Department of Women's and Children's Health, University of Padua, Padua, Italy \\ ${ }^{6}$ Department of Pediatric Hematology/Oncology, Dana-Farber/Boston Children's Cancer and Blood Disorders Center and \\ Harvard Medical School, Boston, MA, USA
}

Correspondence to: Clara Benna, email: clara.benna@unipd.it

Keywords: sarcoma; SNP; meta-analysis; polymorphisms; risk

Received: January 24, $2018 \quad$ Accepted: March 07, $2018 \quad$ Published: April 06, 2018

Copyright: Benna et al. This is an open-access article distributed under the terms of the Creative Commons Attribution License 3.0 (CC BY 3.0), which permits unrestricted use, distribution, and reproduction in any medium, provided the original author and source are credited.

\section{ABSTRACT}

Background: The genetic architecture of bone and soft tissue sarcomas susceptibility is yet to be elucidated. We aimed to comprehensively collect and metaanalyze the current knowledge on genetic susceptibility in these rare tumors.

Methods: We conducted a systematic review and meta-analysis of the evidence on the association between DNA variation and risk of developing sarcomas through searching PubMed, The Cochrane Library, Scopus and Web of Science databases. To evaluate result credibility, summary evidence was graded according to the Venice criteria and false positive report probability (FPRP) was calculated to further validate result noteworthiness. Integrative analysis of genetic and eQTL (expression quantitative trait locus) data was coupled with network and pathway analysis to explore the hypothesis that specific cell functions are involved in sarcoma predisposition.

Results: We retrieved 90 eligible studies comprising 47,796 subjects (cases: $14,358,30 \%$ ) and investigating 1,126 polymorphisms involving 320 distinct genes. Meta-analysis identified 55 single nucleotide polymorphisms (SNPs) significantly associated with disease risk with a high $(\mathrm{N}=9)$, moderate $(\mathrm{N}=38)$ and low $(\mathrm{N}=8)$ level of evidence, findings being classified as noteworthy basically only when the level of evidence was high. The estimated joint population attributable risk for three independent SNPs (rs11599754 of ZNF365/EGR2, rs231775 of CTLA4, and rs454006 of PRKCG) was $37.2 \%$. We also identified 53 SNPs significantly associated with sarcoma risk based on single studies.

Pathway analysis enabled us to propose that sarcoma predisposition might be linked especially to germline variation of genes whose products are involved in the function of the DNA repair machinery.

Conclusions: We built the first knowledgebase on the evidence linking DNA variation to sarcomas susceptibility, which can be used to generate mechanistic hypotheses and inform future studies in this field of oncology. 


\section{INTRODUCTION}

Sarcomas are a family of rare malignant tumors arising from bone and soft tissues with more than 50 different histologies accounting for about $1-2 \%$ of cancers in adults and $15-20 \%$ in children (worldwide incidence: approximately 200,000 cases per year). The pathogenesis of sarcomas is multifactorial including environmental (such as exposure to ionizing radiations or chemical carcinogens) and genetic components, although the disease rarity represents an objective hurdle to the research in this field of investigation. Significant advances have been made in the understanding of the acquired genetic events leading to sarcomagenesis. It has been recognized that three types of somatic DNA alterations, translocations, mutations, and copy number variations, play a key role in these tumors [1]. As a consequence, sarcomas are grouped into two categories: balanced translocation associated sarcomas (BATS) and complex genotype/karyotype sarcomas (CGKS), which are characterized by a stable genome and genomic instability, respectively [2]. A potential therapeutic implication of such genetic taxonomy classification is that some recurrent chromosomal translocations might be exploited for the development of drugs targeting the protein products of fusion oncogenes [1].

Conversely, knowledge on the role of germline DNA variations in sarcomagenesis is sparse and limited. Although a minority of sarcomas arise within well characterized heritable cancer predisposition syndromes (e.g., osteosarcoma and Bloom syndrome, desmoid tumors and familial adenomatous polyposis) [3], the vast majority of sarcomas occur sporadically and the role of the genetic background in their pathogenesis is to be uncovered. Recent advances in molecular high-throughput technology, which conduct of genome wide association studies (GWAS), is accelerating the pace of discovery of sarcoma predisposition loci.

Looking at the already existing international literature, some investigators have meta-analyzed the evidence regarding a handful of SNPs such as XRCC3 rs861539 [4], MDM2 rs2279744 [5, 6], and CTLA4 rs231775 [7]: however, to the best of our knowledge no comprehensive collection of the available data in this field of oncology has been published thus far.

With the present work we systematically reviewed and meta-analyzed the available evidence in this field in order to: 1) provide readers with the first knowledgebase dedicated to the relationship between germline DNA variation and sarcoma risk; 2) identify areas lacking of meaningful information thus helping to inform future studies; and 3) suggest a biological interpretation of current findings utilizing network and pathway analysis [8] after integrating multiple sources of biological data [9].

\section{RESULTS}

\section{Characteristics of the eligible studies}

We identified 90 eligible articles, comprising 47,796 subjects, 14,358 cases and 33,438 controls. The details of the literature search are summarized in Figure 1.

Based on the prevalent ancestry (ie. the race of at least $80 \%$ of the enrolled subjects) the majority of the studies were Asian ( $\mathrm{N}=57$ studies) the rest being Caucasian ( $\mathrm{N}=25$ studies), or mixed ( $\mathrm{N}=8$ studies). Based on study design, half of included studies were population based case-controls studies ( $\mathrm{N}=40$ studies), the remaining were hospital based ( $\mathrm{N}=39$ studies), with a few $(\mathrm{N}=11)$ being mixed or not specified. Two studies were GWAS $[10,11]$

According to histology, the majority of the eligible studies investigated bone tumors $(\mathrm{N}=65)$ and the remaining investigated Ewing's sarcoma $(\mathrm{N}=9)$, soft tissue sarcomas $(\mathrm{N}=6)$, chordoma $(\mathrm{N}=4)$, hemangiosarcoma $(\mathrm{N}=1)$, and mixed sarcomas $(\mathrm{N}=5)$. Thirteen studies investigated pediatric subjects or young adults. Although pediatric/young age ranged from 0 to 35 years old in eligible studies, most of the studies considered subjects $<20$ years old.

We evaluated the included studies following the criteria of the Newcastle-Ottawa scale (NOS) scoring system. The mean score was 7.8. The main features of all the eligible studies and the NOS score are available on Table 1.

\section{Characteristics of the retrieved genetic variants}

Overall, data on 1,126 polymorphisms involving 320 genes were retrieved. Variations were mainly SNPs, only six being insertion/deletions of more than one nucleotide. Based on the number of different genetic variations studied, the 11 most studied genes were the following: EGR2 (179 different SNPs), ADO (58 different SNPs), ZNF365 (40 different SNPs), TRAPPC9 (28 different SNPs), CASC8 (23 different SNPs), CD99 (20 different SNPs), EWSR1 (16 different SNPs) TP53, HSD17B2 (15 different SNPs each) and UGT1A8, LOC107984012 (12 different SNPs each).

Thirty-seven of these genetic variants were located no more than $2 \mathrm{~kb}$ upstream the relevant gene, ten no more than 500bp downstream the relevant gene, 493 in introns, 100 in exons (non-UTRs), 19 in the 3'-UTR, seven in the 5'-UTR. Moreover, 413 SNPs were located in intergenic regions more than $2 \mathrm{~kb}$ upstream or more than $500 \mathrm{bp}$ downstream the relevant gene and 41 in non-coding transcripts. Among the exonic SNPs, 63 had a missense functional effect, while 37 were synonymous. Detailed information on all SNPs is reported in Supplementary Table 1. 


\section{Meta-analysis findings}

At least two independent datasets were available for 51 genetic variations allowing us to perform 118 metaanalyses, 16 of them were histology-based meta-analysis on osteosarcoma and Ewing's sarcoma. Moreover, 13 sensitivity analysis were performed considering the ethnicity of the different datasets. The results of data metaanalyses are comprehensively reported in Supplementary Table 2. Polymorphism "rs" identifier, nucleotide change and amino acid change are reported in Supplementary Table 3 .

The eight most studied genetic variants were the following: TP53 rs 1042522 (6 datasets), VEGF rs3025039 and GSTM1 deletion (5 datasets each), CTLA4 rs231775, CTLA4 rs5742909, MDM2 rs2279744, rs10434 VEGF and GSTT1 deletion (4 datasets each).

The number of subject (cases plus controls) enrolled in the 118 meta-analyses ranged from 144 to 5,347 (median: 1,195). Based on the number of subjects, the 10 most studied genetic variants, all with 5,347 subjects, were the following: EGR2 rs224292 and rs224278, $A D O$ rs1848797 and rs1509966, MDM2 rs1690916, LOC107984012 rs9633562, rs944684 and rs6479860, ZNF365 rs11599754 and rs10761660.

Of the 118 meta-analyses and 13 sensitivity analysis (131 total analyses) performed, 55 resulted to be statistically significant (P-value $<0.05$ ). The level of summary evidence, among the significant associations identified by meta-analysis, was high, intermediate, and low in 9, 38, and 8 analyses respectively. The most frequent single cause of non-high-quality level of evidence was between-study heterogeneity followed by the small sample size. Considering all statistically significant metaanalyses FPRP was optimal $(<0.2)$ at least at the 10E3 level for 10/55 analysis, 9 of them with high level of summary evidence.

The details of significant associations are reported in Table 2.

In order to provide an estimate of the impact of germline variants on sarcoma risk, the PAR (population attributable risk) was calculated. As an example, we considered the following three independent SNPs with high quality evidence on their relationship with sarcoma risk: rs11599754 of ZNF365/EGR2 (chromosome 10, risk allele: $\mathrm{C}$, risk allele frequency in European ancestry population: 0.39 , meta-analysis OR: 1.48 ); rs 231775 of CTLA4 (chromosome 2, risk allele: A, risk allele frequency in European ancestry population: 0.65 , meta-analysis OR: 1.36); and rs454006 of PRKCG (chromosome 19, risk allele: $\mathrm{C}$, risk allele frequency in European ancestry population: 0.25 , meta-analysis OR: 1.35 ). The PAR resulted equal to $37.2 \%$.

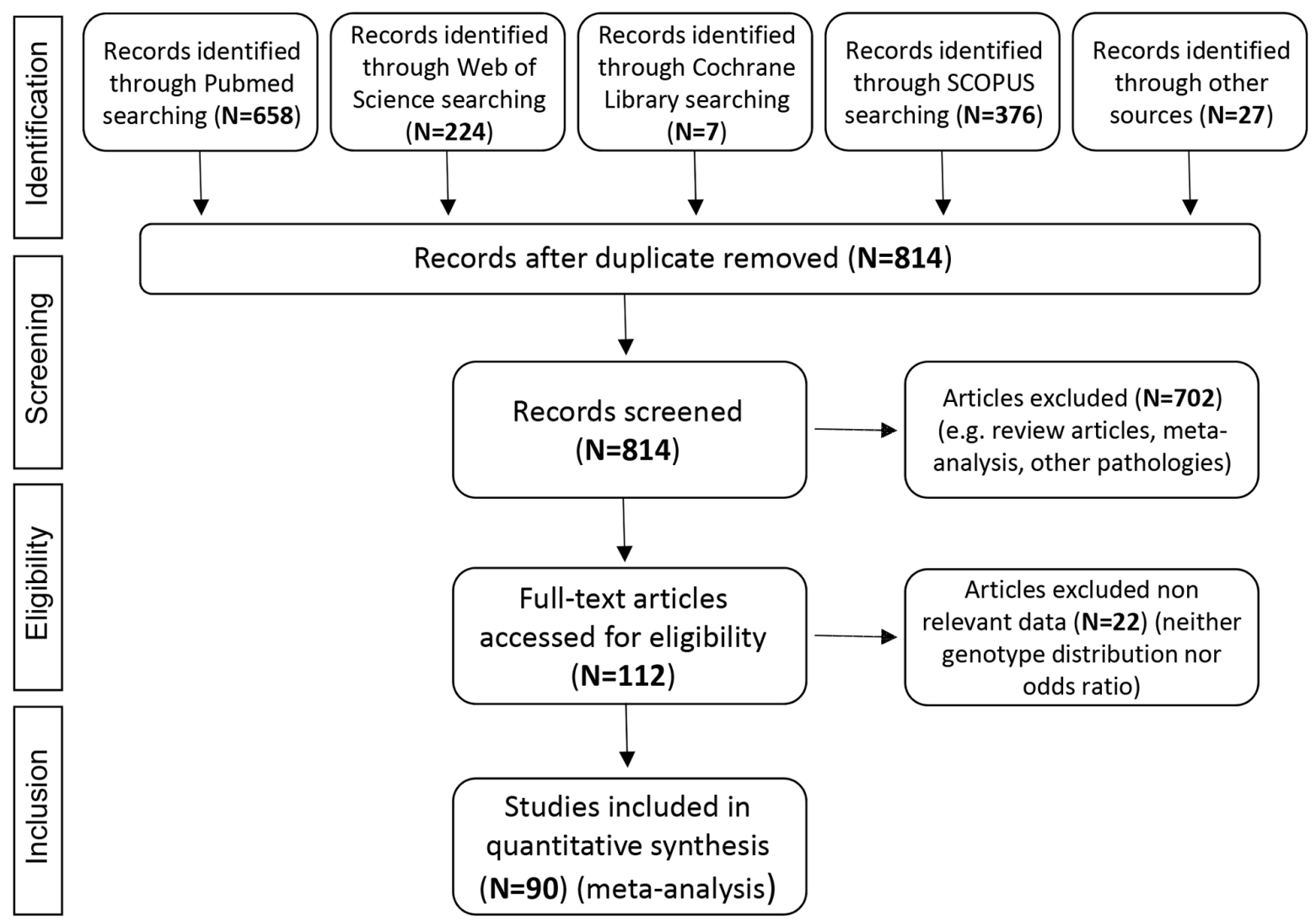

Figure 1: Flow diagram summarizing the search strategy and the study selection process. 
Table 1: Characteristics of the included studies and Newcastle-Ottawa quality assessment (NOS) evaluation

\begin{tabular}{|c|c|c|c|c|c|c|c|c|c|c|}
\hline \multicolumn{3}{|c|}{ Included articles references } & \multicolumn{6}{|c|}{ Subjects characteristics } & \multicolumn{2}{|c|}{ NOS } \\
\hline First Author & Journal & Year & Cancer Type & Cases & Controls & Age & Ethnicity & $\begin{array}{l}\text { Source of } \\
\text { Controls }\end{array}$ & $\begin{array}{l}\text { NOS } \\
123\end{array}$ & $\begin{array}{l}\text { NOS } \\
{[0-9]}\end{array}$ \\
\hline $\begin{array}{l}\text { Adiguzel M. } \\
{[12]}\end{array}$ & Indian J Exp Biol & 2016 & Bone tumors & 54 & 81 & Adult & Caucasian & Population & 413 & 8 \\
\hline $\begin{array}{l}\text { Alhopuro P. } \\
\text { [13] }\end{array}$ & J Med Genet & 2005 & Soft tissue sarcoma & 68 & 185 & Adult & Caucasian & Population & 413 & 8 \\
\hline $\begin{array}{l}\text { Almeida PSR. } \\
\text { [14] }\end{array}$ & Genet Mol Res & 2008 & Soft tissue sarcoma & 100 & 85 & Adult & Mixed & not specified & 213 & 6 \\
\hline $\begin{array}{l}\text { Aoyama T. } \\
{[15]}\end{array}$ & Cancer Letters & 2002 & Bone tumors & 38 & 72 & Adult & Asian & Population & 313 & 7 \\
\hline $\begin{array}{l}\text { Barnette P. } \\
{[16]}\end{array}$ & $\begin{array}{l}\text { Cancer Epidemiol } \\
\text { Biomarkers Prev }\end{array}$ & 2004 & Mixed & 42 & 326 & $\begin{array}{l}\text { Pediat/ } \\
\text { Young }\end{array}$ & Caucasian & Population & 323 & 8 \\
\hline Biason P. [17] & Pharmacogenomics J & 2012 & Bone tumors & 130 & 250 & Adult & Caucasian & Hospital & 323 & 8 \\
\hline $\begin{array}{l}\text { Bilbao- } \\
\text { Aldaiturriaga } \\
\text { N. [18] }\end{array}$ & Pediatr Blood Cancer & 2015 & Bone tumors & 99 & 387 & $\begin{array}{l}\text { Pediat/ } \\
\text { Young }\end{array}$ & Caucasian & Hospital & 323 & 8 \\
\hline Chen Y. [19] & Tumor Biol & 2016 & Bone tumors & 190 & 190 & Adult & Asian & Hospital & 323 & 8 \\
\hline Cong Y. [20] & Tumor Biol & 2015 & Bone tumors & 203 & 406 & Adult & Asian & Hospital & 323 & 8 \\
\hline Cui Y. [21] & Biomarkers & 2016 & Bone tumors & 251 & 251 & Adult & Asian & Hospital & 323 & 8 \\
\hline Cui Y. [22] & Tumor Biol & 2016 & Bone tumors & 260 & 260 & Adult & Asian & Hospital & 323 & 8 \\
\hline Dong YZ. [23] & Genet Mol Res & 2015 & Bone tumors & 185 & 201 & Adult & Asian & Hospital & 323 & 8 \\
\hline $\begin{array}{l}\text { DuBois SG. } \\
\text { [24] }\end{array}$ & Pediatr Blood Cancer & 2011 & Ewing's sarcoma & 135 & 200 & $\begin{array}{l}\text { Pediat/ } \\
\text { Young }\end{array}$ & Caucasian & Hospital & 213 & 6 \\
\hline Ergen A. [25] & Mol Biol Rep & 2011 & Bone tumors & 50 & 50 & Adult & Caucasian & not specified & 313 & 7 \\
\hline Feng D. [26] & $\begin{array}{l}\text { Genet Test Mol } \\
\text { Biomarkers }\end{array}$ & 2013 & Ewing's sarcoma & 308 & 362 & Adult & Asian & Hospital & 323 & 8 \\
\hline $\begin{array}{l}\text { Gloudemans } \\
\text { T. [27] }\end{array}$ & Cancer Res & 1993 & Soft tissue sarcoma & 9 & 26 & Adult & Caucasian & Population & 303 & 6 \\
\hline $\begin{array}{l}\text { Grochola LF. } \\
{[28]}\end{array}$ & Clin Cancer Res & 2009 & Soft tissue sarcoma & 130 & 497 & Adult & Caucasian & Population & 313 & 7 \\
\hline $\begin{array}{l}\text { Grünewald } \\
\text { TG. [29] }\end{array}$ & Nat Genet & 2015 & Ewing's sarcoma & 343 & 251 & Adult & Caucasian & Population & 423 & 9 \\
\hline Guo J. [30] & Genet Mol Res & 2015 & Bone tumors & 136 & 136 & Adult & Asian & Hospital & 313 & 7 \\
\hline He J. [31] & Endocr J & 2013 & Bone tumors & 415 & 431 & Adult & Asian & Hospital & 323 & 8 \\
\hline He J. [32] & Endocrine & 2014 & Bone tumors & 415 & 431 & Adult & Asian & Hospital & 323 & 8 \\
\hline He M. [33] & Tumor Biol & 2014 & Bone tumors & 189 & 195 & Adult & Asian & Hospital & 323 & 8 \\
\hline He ML. [34] & $\begin{array}{c}\text { Asian Pac J Cancer } \\
\text { Prev }\end{array}$ & 2013 & Bone tumors & 59 & 63 & Adult & Asian & Hospital & 313 & 7 \\
\hline He Y. [35] & Int Orthop & 2014 & Bone tumors & 120 & 120 & Adult & Asian & Hospital & 323 & 8 \\
\hline Hu GL. [36] & Genet Mol Res & 2015 & Bone tumors & 130 & 130 & Adult & Asian & Hospital & 323 & 8 \\
\hline Hu YS. [37] & BMC Cancer & 2010 & Bone tumors & 168 & 168 & Adult & Asian & Population & 423 & 9 \\
\hline Hu YS. [38] & Med Oncol & 2011 & Bone tumors & 168 & 168 & Adult & Asian & Population & 423 & 9 \\
\hline Hu Z. [39] & $\begin{array}{l}\text { Genet Test Mol } \\
\text { Biomarkers }\end{array}$ & 2015 & Bone tumors & 368 & 370 & Adult & Asian & not specified & 213 & 6 \\
\hline Ito M. [40] & Clin Cancer Res & 2010 & Soft tissue sarcoma & 155 & 37 & Adult & Mixed & Hospital & 203 & 5 \\
\hline Jiang C. [41] & Med Oncol & 2014 & Bone tumors & 168 & 216 & Adult & Asian & Hospital & 323 & 8 \\
\hline $\begin{array}{l}\text { Kelley MJ. } \\
{[42]}\end{array}$ & Hum Genet & 2014 & Chordoma & 103 & 160 & Adult & Asian & Population & 413 & 8 \\
\hline $\begin{array}{l}\text { Koshkina NV. } \\
{[43]}\end{array}$ & $\begin{array}{l}\text { J Pediatr Hematol } \\
\text { Oncol }\end{array}$ & 2007 & Bone tumors & 123 & 510 & $\begin{array}{l}\text { Pediat/ } \\
\text { Young }\end{array}$ & Mixed & Population & 413 & 8 \\
\hline $\begin{array}{l}\text { Le Morvan V. } \\
\text { [44] }\end{array}$ & Int J Cancer & 2006 & Mixed & 93 & 53 & Adult & Caucasian & Population & 403 & 7 \\
\hline
\end{tabular}




\begin{tabular}{|c|c|c|c|c|c|c|c|c|c|c|}
\hline \multicolumn{3}{|c|}{ Included articles references } & \multicolumn{6}{|c|}{ Subjects characteristics } & \multicolumn{2}{|c|}{ NOS } \\
\hline First Author & Journal & Year & Cancer Type & Cases & Controls & Age & Ethnicity & $\begin{array}{l}\text { Source of } \\
\text { Controls }\end{array}$ & $\begin{array}{c}\text { NOS } \\
123\end{array}$ & $\begin{array}{l}\text { NOS } \\
{[0-9]}\end{array}$ \\
\hline Li L. [45] & Genet Mol Res & 2015 & Bone tumors & 52 & 100 & Adult & Asian & Hospital & 312 & 6 \\
\hline Liu Y. [46] & DNA Cell Biol & 2011 & Bone tumors & 267 & 282 & Adult & Asian & Population & 313 & 7 \\
\hline Liu Y. [47] & PloSONE & 2012 & Bone tumors & 326 & 433 & Adult & Asian & Population & 423 & 9 \\
\hline Lu H. [48] & Tumor Biol & 2015 & Bone tumors & 388 & 388 & Adult & Asian & Hospital & 323 & 8 \\
\hline Lu XF. [49] & $\begin{array}{c}\text { Asian Pac J Cancer } \\
\text { Prev }\end{array}$ & 2011 & Bone tumors & 110 & 226 & Adult & Asian & Hospital & 313 & 7 \\
\hline Lv H. [50] & Mol Med Rep & 2014 & Bone tumors & 103 & 201 & Adult & Asian & Hospital & 213 & 6 \\
\hline Ma X. [51] & Genet Mol Res & 2016 & Bone tumors & 141 & 282 & Adult & Asian & Hospital & 223 & 7 \\
\hline $\begin{array}{l}\text { Martinelli M. } \\
\text { [52] }\end{array}$ & Oncotarget & 2016 & Ewing's sarcoma & 100 & 147 & $\begin{array}{l}\text { Pediat/ } \\
\text { Young }\end{array}$ & Caucasian & Population & 423 & 9 \\
\hline Mei JW. [99] & Int J Clin Exp Pathol & 2016 & Bone tumors & 97 & 120 & Adult & Asian & Population & 313 & 7 \\
\hline Miao C.[53] & Sci Rep & 2015 & Soft tissue sarcoma & 138 & 131 & Adult & Asian & Hospital & 223 & 7 \\
\hline $\begin{array}{l}\text { Mirabello L. } \\
{[54]}\end{array}$ & Carcinogenesis & 2010 & Bone tumors & 99 & 1430 & Adult & Caucasian & mixed & 323 & 8 \\
\hline $\begin{array}{l}\text { Mirabello L. } \\
{[55]}\end{array}$ & BMC Cancer & 2011 & Bone tumors & 96 & 1426 & Adult & Caucasian & mixed & 323 & 8 \\
\hline $\begin{array}{l}\text { Nakayama R. } \\
{[56]}\end{array}$ & Cancer Sci & 2008 & Mixed & 544 & 1378 & Adult & Asian & mixed & 323 & 8 \\
\hline $\begin{array}{l}\text { Naumov VA. } \\
\text { [57] }\end{array}$ & Bull Exp Biol Med & 2012 & Bone tumors & 68 & 96 & Adult & Caucasian & not specified & 313 & 7 \\
\hline $\begin{array}{l}\text { Oliveira ID. } \\
\text { [58] }\end{array}$ & $\begin{array}{l}\text { J Pediatr Hematol } \\
\text { Oncol }\end{array}$ & 2007 & Bone tumors & 80 & 160 & $\begin{array}{l}\text { Pediat/ } \\
\text { Young }\end{array}$ & Mixed & Hospital & 323 & 8 \\
\hline Ozger H. [59] & Folia Biologica (Praha) & 2008 & Mixed & 56 & 44 & Adult & Caucasian & Population & 403 & 7 \\
\hline $\begin{array}{l}\text { Patino-Garcia } \\
\text { A. [60] }\end{array}$ & J Med Genet & 2000 & Bone tumors & 110 & 111 & $\begin{array}{l}\text { Pediat/ } \\
\text { Young }\end{array}$ & Caucasian & not specified & 323 & 8 \\
\hline Pillay N. [61] & Nat Genet & 2012 & Chordoma & 40 & 358 & Adult & Caucasian & population & 323 & 8 \\
\hline $\begin{array}{l}\text { Postel-Vinay } \\
\text { S. [10] }\end{array}$ & Nat Genet & 2012 & Ewing's sarcoma & 401 & 4352 & Adult & Caucasian & population & 423 & 9 \\
\hline Qi Y. [62] & Tumor Biol & 2016 & Bone tumors & 206 & 206 & Adult & Asian & Hospital & 323 & 8 \\
\hline Qu WR. [63] & Genetic Mol Res & 2016 & Bone tumors & 153 & 252 & Adult & Asian & Hospital & 323 & 8 \\
\hline Ru JY. [64] & Int J Clin Exp Pathol & 2015 & Bone tumors & 210 & 420 & Adult & Asian & Hospital & 323 & 8 \\
\hline Ruza E. [65] & $\begin{array}{l}\text { J Pediatr Hematol } \\
\text { Oncol }\end{array}$ & 2003 & Mixed & 125 & 143 & $\begin{array}{l}\text { Pediat/ } \\
\text { Young }\end{array}$ & Caucasian & not specified & 322 & 7 \\
\hline Saito T. [66] & Int J Cancer & 2000 & Hemangiosarcoma & 22 & 84 & Adult & Mixed & Population & 213 & 6 \\
\hline $\begin{array}{l}\text { Salinas-Souza } \\
\text { C. [67] }\end{array}$ & $\begin{array}{l}\text { Pharmacogenet } \\
\text { Genomics }\end{array}$ & 2010 & Bone tumors & 80 & 160 & $\begin{array}{l}\text { Pediat/ } \\
\text { Young }\end{array}$ & Mixed & Hospital & 323 & 8 \\
\hline $\begin{array}{l}\text { Savage SA. } \\
{[68]}\end{array}$ & $\begin{array}{l}\text { Cancer Epidemiol } \\
\text { Biomarkers Prev }\end{array}$ & 2007 & Bone tumors & 104 & 74 & $\begin{array}{l}\text { Pediat/ } \\
\text { Young }\end{array}$ & Caucasian & Hospital & 213 & 6 \\
\hline $\begin{array}{l}\text { Savage SA. } \\
{[69]}\end{array}$ & Pediatr Blood Cancer & 2007 & Bone tumors & 104 & 74 & $\begin{array}{l}\text { Pediat/ } \\
\text { Young }\end{array}$ & Caucasian & Hospital & 213 & 6 \\
\hline $\begin{array}{l}\text { Savage SA. } \\
{[11]}\end{array}$ & Nat Genet & 2013 & Bone tumors & 941 & 3291 & Adult & Caucasian & Population & 423 & 9 \\
\hline Shi ZW. [70] & Cancer Biomark & 2016 & Bone tumors & 174 & 150 & Adult & Asian & Hospital & 313 & 7 \\
\hline Silva DS. [71] & Gene & 2012 & Ewing's sarcoma & 24 & 200 & Adult & Mixed & Population & 323 & 8 \\
\hline Tang YJ. [72] & Medicine & 2014 & Bone tumors & 160 & 250 & Adult & Asian & Population & 423 & 9 \\
\hline $\begin{array}{l}\text { Thurow HS. } \\
\text { [73] }\end{array}$ & Mol Biol Rep & 2013 & Ewing's sarcoma & 24 & 91 & Adult & Mixed & Population & 323 & 8 \\
\hline Tian Q. [74] & Eur J Surg Oncol & 2013 & Bone tumors & 133 & 133 & Adult & Asian & Population & 423 & 9 \\
\hline Tie Z. [75] & Int J Clin Exp Pathol & 2014 & Bone tumors & 165 & 330 & Adult & Asian & Population & 423 & 9 \\
\hline Toffoli G. [76] & Clin Cancer Res & 2009 & Bone tumors & 201 & 250 & Adult & Caucasian & Population & 423 & 9 \\
\hline
\end{tabular}




\begin{tabular}{|c|c|c|c|c|c|c|c|c|c|c|}
\hline \multicolumn{3}{|c|}{ Included articles references } & \multicolumn{6}{|c|}{ Subjects characteristics } & \multicolumn{2}{|c|}{ NOS } \\
\hline First Author & Journal & Year & Cancer Type & Cases & Controls & Age & Ethnicity & $\begin{array}{l}\text { Source of } \\
\text { Controls }\end{array}$ & $\begin{array}{l}\text { NOS } \\
123\end{array}$ & $\begin{array}{l}\text { NOS } \\
{[0-9]}\end{array}$ \\
\hline $\begin{array}{l}\text { Walsh KM. } \\
\text { [77] }\end{array}$ & Carcinogenesis & 2016 & Bone tumors & 660 & 6892 & $\begin{array}{l}\text { Pediat/ } \\
\text { Young }\end{array}$ & Caucasian & Population & 423 & 9 \\
\hline Wang J. [78] & DNA Cell Biol & 2012 & Ewing's sarcoma & 158 & 212 & Adult & Asian & Population & 323 & 8 \\
\hline Wang J. [79] & DNA Cell Biol & 2013 & Bone tumors & 106 & 210 & Adult & Asian & Population & 323 & 8 \\
\hline Wang K. [80] & Biomed Rep & 2014 & Chordoma & 65 & 65 & Adult & Asian & Population & 313 & 7 \\
\hline Wang K. [81] & Tumor Biol & 2016 & Bone tumors & 126 & 168 & Adult & Asian & Hospital & 323 & 8 \\
\hline Wang W. [82] & DNA Cell Biol & 2011 & Bone tumors & 205 & 216 & Adult & Asian & Hospital & 323 & 8 \\
\hline Wang W. [83] & $\begin{array}{c}\text { Genet Test Mol } \\
\text { Biomarkers }\end{array}$ & 2011 & Bone tumors & 205 & 215 & Adult & Asian & Hospital & 323 & 8 \\
\hline Wang Z. [84] & Tumor Biol & 2014 & Bone tumors & 330 & 342 & Adult & Asian & Population & 423 & 9 \\
\hline Wu Y. [85] & Tumor Biol & 2015 & Bone tumors & 124 & 136 & Adult & Asian & Hospital & 323 & 8 \\
\hline Wu Z. [86] & Int J Mol Sci & 2013 & Chordoma & 65 & 120 & Adult & Asian & not specified & 313 & 7 \\
\hline Xin DJ. [87] & Int J Clin Exp Pathol & 2015 & Bone tumors & 90 & 100 & Adult & Asian & Population & 413 & 8 \\
\hline Xu H. [88] & Med Sci Monit & 2016 & Bone tumors & 279 & 286 & $\begin{array}{l}\text { Pediat/ } \\
\text { Young }\end{array}$ & Asian & Hospital & 323 & 8 \\
\hline Xu S. [89] & DNA Cell Biol & 2014 & Bone tumors & 202 & 216 & Adult & Asian & Population & 423 & 9 \\
\hline Yang L. [90] & Int J Clin Exp Pathol & 2015 & Bone tumors & 152 & 304 & Adult & Asian & Population & 423 & 9 \\
\hline Yang S. [91] & $\begin{array}{c}\text { Genet Test Mol } \\
\text { Biomarkers }\end{array}$ & 2012 & Ewing's sarcoma & 223 & 302 & Adult & Asian & Population & 423 & 9 \\
\hline Yang W. [92] & Med Oncol & 2014 & Bone tumors & 118 & 126 & Adult & Asian & not specified & 323 & 8 \\
\hline Zhang G. [93] & Genet Mol Res & 2015 & Bone tumors & 180 & 360 & Adult & Asian & Population & 423 & 9 \\
\hline $\begin{array}{l}\text { Zhang HF. } \\
\text { [94] }\end{array}$ & Genet Mol Res & 2015 & Bone tumors & 182 & 182 & Adult & Asian & Population & 423 & 9 \\
\hline Zhang N. [95] & Onco Targets Ther & 2016 & Bone tumors & 276 & 286 & Adult & Asian & Hospital & 323 & 8 \\
\hline Zhang Y. [96] & Tumor Biol & 2014 & Bone tumors & 610 & 610 & Adult & Asian & Population & 423 & 9 \\
\hline Zhao J. [97] & BioMed Res Int & 2014 & Bone tumors & 247 & 428 & Adult & Asian & Population & 423 & 9 \\
\hline Zhi LQ. [98] & Tumor Biol & 2014 & Bone tumors & 212 & 240 & Adult & Asian & Hospital & 323 & 8 \\
\hline
\end{tabular}

NOS: Newcastle-Ottawa quality assessment scale evaluation (0-9). NOS1: selection of the study groups (0-4); NOS2: comparability of the groups (0-2); NOS3: ascertainment of the exposure or outcome (0-3).

\section{Associations based on single studies}

Beside the variations resulted to be statistically significantly associated with sarcoma risk in this metaanalysis, we retrieved from the included articles 906 SNPs statistically significantly associated with sarcoma risk (P-value $<0.05$ ) based on single-study analysis. In Table 3 are reported 53 SNPs strongly associated with Ewing's sarcoma or osteosarcoma risk (P-value $<\mathrm{E}-06$ ), retrieved from the included studies.

One dataset was available for each of those genetic variants. Although it was not possible to perform a metaanalysis, a strong association with sarcoma risk was found (P-values range from E-20 to E-06). Ewing's sarcoma associations in European and US European-descendant population mainly involved the candidate risk loci at 1p36.22, 10q21 reported by Postel-Vinay et al [10] GWAS and in the following related study of Grünewald et al [29]. The $1 \mathrm{p} 36.22$ variants associated with Ewing's sarcoma are located $25 \mathrm{~kb}$ proximal to the TARDBP gene. TARDBP (Tat activating regulatory DNA-binding protein, or TDP43 , transactive response DNA-binding protein) is a highly conserved DNA- and RNA-binding protein involved in RNA transcription and splicing. The 10q21 variants strongly associated with Ewing's sarcoma are located in a block containing four genes: $A D O$ (encoding cysteamine dioxygenase), ZNF365 (encoding zinc-finger protein 365), $E G R 2$ (encoding early growth response protein 2) and LOC107984012 (unknown function).

A further association with osteosarcoma in Guangxi population was studied by Zhao et al [97] regarding the Rho GTPase-activating protein 35 (ARHGAP35), a Rho family GTPase-activating protein. Finally Savage et al [11] GWAS found associations with osteosarcoma and 
Table 2: Meta-analysis results: genetic variants significantly associated with sarcoma risk

\begin{tabular}{|c|c|c|c|c|c|c|c|c|c|c|c|c|c|c|c|}
\hline SNP ID & Genes & Analysis & Model & $\begin{array}{l}\text { Sarcoma } \\
\text { type }\end{array}$ & $\begin{array}{l}\text { data } \\
\text { sets }\end{array}$ & $\begin{array}{c}\text { Meta- } \\
\text { analysis } \\
\text { Ethnicity }\end{array}$ & OR $[95 \% \mathrm{CI}]$ & $I^{2} \%$ & P value & Cases & Controls & $\begin{array}{l}\text { Ref/ } \\
\text { Alt }\end{array}$ & $\begin{array}{l}\text { Venice } \\
\text { Criteria }\end{array}$ & $\begin{array}{l}\text { FPRP } \\
(\mathbf{E - 0 3 )}\end{array}$ & $\begin{array}{c}\text { Level of } \\
\text { Evidence }\end{array}$ \\
\hline rs11599754 & ZNF365, ADO & primary & Per allele & Ewing's & 2 & Caucasian & $1.48[1.32,1.66]$ & 0 & $<0.00001$ & 744 & 4603 & $\mathrm{~T} / \mathrm{C}$ & AAA & Y & HIGH \\
\hline rs1509966 & ADO, EGR2 & primary & Per allele & Ewing's & 2 & Caucasian & $1.58[1.42,1.77]$ & 0 & $<0.00001$ & 744 & 4603 & $\mathrm{~A} / \mathrm{G}$ & AAA & $\mathrm{Y}$ & HIGH \\
\hline rs 1848797 & ADO, EGR2 & primary & Per allele & Ewing's & 2 & Caucasian & $1.57[1.4,1.77]$ & 0 & $<0.00001$ & 744 & 4603 & $\mathrm{G} / \mathrm{A}$ & AAA & $\mathrm{Y}$ & HIGH \\
\hline rs 224278 & EGR2 & primary & Per allele & Ewing's & 2 & Caucasian & $1.73[1.49,2.02]$ & 0 & $<0.00001$ & 744 & 4603 & $\mathrm{~T} / \mathrm{C}$ & AAA & $\mathrm{Y}$ & HIGH \\
\hline rs9633562 & $\begin{array}{c}\text { EGR2, } \\
\text { LOC107984012 }\end{array}$ & primary & Per allele & Ewing's & 2 & Caucasian & $1.46[1.29,1.65]$ & 0 & $<0.00001$ & 744 & 4603 & $\mathrm{~A} / \mathrm{C}$ & AAA & $\mathrm{Y}$ & HIGH \\
\hline rs10761660 & ADO, EGR2 & primary & Per allele & Ewing's & 2 & Caucasian & $1.39[1.21,1.6]$ & 0 & $<0.00001$ & 744 & 4603 & $\mathrm{~T} / \mathrm{C}$ & AAA & $\mathrm{Y}$ & HIGH \\
\hline rs 224292 & ADO, EGR2 & primary & Per allele & Ewing's & 2 & Caucasian & $1.67[1.42,1.96]$ & 0 & $<0.00001$ & 744 & 4603 & $\mathrm{~A} / \mathrm{G}$ & AAA & $\mathrm{Y}$ & HIGH \\
\hline rs 231775 & CTLA4 & primary & Per allele & Mixed & 4 & Asian & $1.36[1.2,1.54]$ & 0 & $<0.00001$ & 1003 & 1162 & $\mathrm{G} / \mathrm{A}$ & AAA & Y & HIGH \\
\hline rs 454006 & PRKCG & primary & Per allele & Osteo & 2 & Asian & $1.35[1.18,1.54]$ & 0 & $<0.0001$ & 998 & 998 & $\mathrm{~T} / \mathrm{C}$ & AAA & $\mathrm{Y}$ & HIGH \\
\hline rs 944684 & LOC107984012 & primary & Per allele & Ewing's & 2 & Caucasian & $1.73[1.4,2.14]$ & 49 & $<0.00001$ & 744 & 4603 & $\mathrm{C} / \mathrm{T}$ & $\mathrm{ABA}$ & $\mathrm{Y}$ & INTERM \\
\hline rs2305089 & $\mathrm{T}$ & sensitivity & Per allele & Chordoma & 2 & Caucasian & $3.91[2.4,6.38]$ & 47 & $<0.00001$ & 163 & 881 & $\mathrm{G} / \mathrm{A}$ & $\mathrm{ABA}$ & $\mathrm{N}$ & INTERM \\
\hline rs 1042522 & ТР53 & primary & Dominant & Mixed & 6 & Mixed & $0.67[0.53,0.84]$ & 0 & 0.0007 & 788 & 950 & $\mathrm{G} / \mathrm{C}$ & AAA & $\mathrm{N}$ & INTERM \\
\hline rs 1042522 & TP53 & subgroup & Dominant & Osteo & 3 & Mixed & $0.6[0.43,0.84]$ & 15 & 0.002 & 509 & 737 & $\mathrm{G} / \mathrm{C}$ & AAA & $\mathrm{N}$ & INTERM \\
\hline rs 1129055 & CD86 & primary & Recessive & Mixed & 2 & Asian & $0.6[0.41,0.88]$ & 0 & 0.008 & 363 & 428 & $\mathrm{~A} / \mathrm{G}$ & BAA & $\mathrm{N}$ & INTERM \\
\hline rs11737764 & NUDT6 & primary & Dominant & Bone tumor & 2 & Caucasian & $2.12[1.34,3.37]$ & 0 & 0.001 & 164 & 1522 & $\mathrm{~A} / \mathrm{C}$ & AAA & $\mathrm{N}$ & INTERM \\
\hline rs1690916 & MDM2 & primary & Per allele & Ewing's & 2 & Caucasian & $0.62[0.46,0.83]$ & 0 & 0.001 & 164 & 1522 & $\mathrm{C} / \mathrm{T}$ & AAA & $\mathrm{N}$ & INTERM \\
\hline rs17206779 & ADAMTS6 & primary & Per allele & Osteo & 2 & Mixed & $0.79[0.67,0.93]$ & 35 & 0.004 & 1109 & 3507 & $\mathrm{C} / \mathrm{T}$ & $\mathrm{ABA}$ & $\mathrm{N}$ & INTERM \\
\hline rs 17655 & ERCC5 & primary & Recessive & Mixed & 2 & Caucasian & $2.04[1.07,3.9]$ & 0 & 0.03 & 223 & 515 & $\mathrm{G} / \mathrm{C}$ & BAA & $\mathrm{N}$ & INTERM \\
\hline rs1799793 & ERCC2 & primary & Per allele & Osteo & 2 & Mixed & $0.75[0.58,0.97]$ & 23 & 0.03 & 271 & 532 & $\mathrm{G} / \mathrm{A}$ & BAA & $\mathrm{N}$ & INTERM \\
\hline rs1799793 & ERCC2 & primary & Dominant & Osteo & 2 & Mixed & $0.63[0.44,0.89]$ & 0 & 0.009 & 271 & 532 & $\mathrm{G} / \mathrm{A}$ & BAA & $\mathrm{N}$ & INTERM \\
\hline rs1800896 & IL10 & primary & Per allele & Osteo & 2 & Mixed & $1.33[1.06,1.66]$ & 0 & 0.01 & 340 & 420 & $\mathrm{~A} / \mathrm{G}$ & BAA & $\mathrm{N}$ & INTERM \\
\hline rs1906953 & GRM4 & sensitivity & Per allele & Osteo & 2 & Asian & $0.68[0.55,0.84]$ & 0 & 0.0004 & 294 & 384 & $\mathrm{G} / \mathrm{A}$ & BAA & $\mathrm{N}$ & INTERM \\
\hline rs2279744 & MDM2 & primary & Per allele & Mixed & 4 & Mixed & $1.36[1.06,1.76]$ & 26 & 0.02 & 448 & 563 & $\mathrm{~T} / \mathrm{G}$ & $\mathrm{ABA}$ & $\mathrm{N}$ & INTERM \\
\hline rs2279744 & MDM2 & primary & Recessive & Mixed & 4 & Mixed & $1.58[1.03,2.42]$ & 20 & 0.04 & 448 & 563 & $\mathrm{~T} / \mathrm{G}$ & AAA & $\mathrm{N}$ & INTERM \\
\hline rs2279744 & MDM2 & primary & Dominant & Mixed & 4 & Mixed & $1.55[1.05,2.29]$ & 36 & 0.03 & 448 & 563 & $\mathrm{~T} / \mathrm{G}$ & $\mathrm{ABA}$ & $\mathrm{N}$ & INTERM \\
\hline rs 231775 & CTLA4 & primary & Recessive & Mixed & 4 & Asian & $2[1.53,2.62]$ & 0 & $<0.00001$ & 1003 & 1162 & $\mathrm{G} / \mathrm{A}$ & AAA & $\mathrm{N}$ & INTERM \\
\hline rs 231775 & CTLA4 & primary & Dominant & Mixed & 4 & Asian & $1.35[1.14,1.61]$ & 0 & 0.0007 & 1003 & 1162 & $\mathrm{G} / \mathrm{A}$ & AAA & $\mathrm{N}$ & INTERM \\
\hline rs 231775 & CTLA4 & subgroup & Per allele & Ewing's & 2 & Asian & $1.36[1.15,1.61]$ & 0 & 0.0003 & 531 & 664 & $\mathrm{G} / \mathrm{A}$ & AAA & $\mathrm{N}$ & INTERM \\
\hline rs231775 & CTLA4 & subgroup & Recessive & Ewing's & 2 & Asian & $2[1.39,2.89]$ & 0 & 0.0002 & 531 & 664 & $\mathrm{G} / \mathrm{A}$ & AAA & $\mathrm{N}$ & INTERM \\
\hline rs 231775 & CTLA4 & subgroup & Dominant & Ewing's & 2 & Asian & $1.36[1.07,1.72]$ & 0 & 0.01 & 531 & 664 & $\mathrm{G} / \mathrm{A}$ & AAA & $\mathrm{N}$ & INTERM \\
\hline rs 231775 & CTLA4 & subgroup & Per allele & Osteo & 2 & Asian & $1.36[1.13,1.64]$ & 0 & 0.001 & 472 & 498 & $\mathrm{G} / \mathrm{A}$ & $\mathrm{ABA}$ & $\mathrm{N}$ & INTERM \\
\hline rs 231775 & CTLA4 & subgroup & Recessive & Osteo & 2 & Asian & $2[1.34,2.98]$ & 0 & 0.0007 & 472 & 498 & $\mathrm{G} / \mathrm{A}$ & $\mathrm{ABA}$ & $\mathrm{N}$ & INTERM \\
\hline rs 231775 & CTLA4 & subgroup & Dominant & Osteo & 2 & Asian & $1.35[1.04,1.75]$ & 0 & 0.02 & 472 & 498 & $\mathrm{G} / \mathrm{A}$ & $\mathrm{ABA}$ & $\mathrm{N}$ & INTERM \\
\hline rs3025039 & VEGFA & primary & Per allele & Osteo & 5 & Asian & $1.28[1.12,1.47]$ & 0 & 0.0004 & 987 & 1344 & $\mathrm{C} / \mathrm{T}$ & AAA & $\mathrm{N}$ & INTERM \\
\hline rs3025039 & VEGFA & primary & Recessive & Osteo & 5 & Asian & $1.65[1.19,2.27]$ & 6 & 0.002 & 987 & 1344 & $\mathrm{C} / \mathrm{T}$ & AAA & $\mathrm{N}$ & INTERM \\
\hline rs3025039 & VEGFA & primary & Dominant & Osteo & 5 & Asian & $1.24[1.04,1.47]$ & 0 & 0.02 & 987 & 1344 & $\mathrm{C} / \mathrm{T}$ & AAA & $\mathrm{N}$ & INTERM \\
\hline rs 454006 & PRKCG & primary & Recessive & Osteo & 2 & Asian & $1.99[1.54,2.58]$ & 0 & $<0.0001$ & 998 & 998 & $\mathrm{~T} / \mathrm{C}$ & AAA & $\mathrm{N}$ & INTERM \\
\hline rs6599400 & FGFR3 & primary & Per allele & Osteo & 2 & Caucasian & $1.53[1.19,1.97]$ & 0 & 0.001 & 164 & 1522 & $\mathrm{C} / \mathrm{A}$ & AAA & $\mathrm{N}$ & INTERM \\
\hline rs699947 & VEGFA & primary & Per allele & Osteo & 2 & Asian & $1.46[1.19,1.79]$ & 0 & 0.0003 & 347 & 512 & $\mathrm{C} / \mathrm{A}$ & BAA & $\mathrm{N}$ & INTERM \\
\hline rs699947 & VEGFA & primary & Recessive & Osteo & 2 & Asian & $1.73[1.17,2.55]$ & 0 & 0.006 & 347 & 512 & $\mathrm{C} / \mathrm{A}$ & BAA & $\mathrm{N}$ & INTERM \\
\hline rs699947 & VEGFA & primary & Dominant & Osteo & 2 & Asian & $1.51[1.14,2]$ & 0 & 0.004 & 347 & 512 & $\mathrm{C} / \mathrm{A}$ & BAA & $\mathrm{N}$ & INTERM \\
\hline rs820196 & RECQL5 & primary & Recessive & Osteo & 2 & Asian & $2.15[1.41,3.29]$ & 0 & 0.0004 & 397 & 441 & $\mathrm{~T} / \mathrm{C}$ & BAA & $\mathrm{N}$ & INTERM \\
\hline rs820196 & RECQL5 & primary & Dominant & Osteo & 2 & Asian & $1.49[1.12,1.98]$ & 0 & 0.006 & 397 & 441 & $\mathrm{~T} / \mathrm{C}$ & BAA & $\mathrm{N}$ & INTERM \\
\hline rs861539 & XRCC3, KLC1 & primary & Per allele & Osteo & 2 & Asian & $1.57[1.25,1.97]$ & 0 & 0.0001 & 288 & 440 & $\mathrm{C} / \mathrm{T}$ & BAA & $\mathrm{N}$ & INTERM \\
\hline rs861539 & XRCC3, KLC1 & primary & Recessive & Osteo & 2 & Asian & $2.23[1.4,3.57]$ & 0 & 0.0008 & 288 & 440 & $\mathrm{C} / \mathrm{T}$ & BAA & $\mathrm{N}$ & INTERM \\
\hline rs861539 & XRCC3, KLC1 & primary & Dominant & Osteo & 2 & Asian & $1.57[1.16,2.13]$ & 0 & 0.003 & 288 & 440 & $\mathrm{C} / \mathrm{T}$ & BAA & $\mathrm{N}$ & INTERM \\
\hline deletion & GSTT1 & primary & Recessive & Mixed & 4 & Mixed & $1.32[1.01,1.73]$ & 4 & 0.04 & 355 & 938 & $\begin{array}{l}\text { non- } \\
\text { null/ } \\
\text { null }\end{array}$ & AAA & $\mathrm{N}$ & INTERM \\
\hline rs 1042522 & TP53 & primary & Per allele & Mixed & 6 & Mixed & $0.6[0.39,0.93]$ & 84 & 0.02 & 788 & 950 & $\mathrm{G} / \mathrm{C}$ & ACA & $\mathrm{N}$ & LOW \\
\hline rs 1042522 & TP53 & subgroup & Per allele & Osteo & 3 & Mixed & $0.47[0.23,0.95]$ & 93 & 0.04 & 509 & 737 & $\mathrm{G} / \mathrm{C}$ & $\mathrm{ACA}$ & $\mathrm{N}$ & LOW \\
\hline rs 1129055 & CD86 & primary & Per allele & Mixed & 2 & Asian & $0.33[0.11,1.01]$ & 93 & 0.05 & 363 & 428 & $\mathrm{~A} / \mathrm{G}$ & $\mathrm{BCA}$ & $\mathrm{N}$ & LOW \\
\hline
\end{tabular}




\begin{tabular}{|c|c|c|c|c|c|c|c|c|c|c|c|c|c|c|c|}
\hline SNP ID & Genes & Analysis & Model & $\begin{array}{c}\text { Sarcoma } \\
\text { type }\end{array}$ & $\begin{array}{l}\text { data } \\
\text { sets }\end{array}$ & $\begin{array}{c}\text { Meta- } \\
\text { analysis } \\
\text { Ethnicity }\end{array}$ & OR $[95 \% \mathrm{CI}]$ & $I^{2} \%$ & Pvalue & Cases & Controls & $\begin{array}{c}\text { Ref/ } \\
\text { Alt }\end{array}$ & $\begin{array}{c}\text { Venice } \\
\text { Criteria }\end{array}$ & $\begin{array}{l}\text { FPRP } \\
\text { (E-03) }\end{array}$ & $\begin{array}{l}\text { Level of } \\
\text { Evidence }\end{array}$ \\
\hline rs 2305089 & $\mathrm{~T}$ & primary & Per allele & Chordoma & 3 & Mixed & $2.87[1.35,6.08]$ & 86 & 0.006 & 228 & 1001 & $\mathrm{G} / \mathrm{A}$ & $\mathrm{ACA}$ & $\mathrm{N}$ & LOW \\
\hline rs 2305089 & $\mathrm{~T}$ & primary & Recessive & Chordoma & 2 & Mixed & $4.16[1.21,14.25]$ & 82 & 0.02 & 125 & 841 & $\mathrm{G} / \mathrm{A}$ & $\mathrm{BCA}$ & $\mathrm{N}$ & LOW \\
\hline rs 6479860 & $\begin{array}{l}\text { LOC107984012 } \\
\text { NRBF2 }\end{array}$ & primary & Per allele & Ewing's & 2 & Caucasian & $1.79[1.36,2.34]$ & 66 & $<0.0001$ & 744 & 4603 & $\mathrm{C} / \mathrm{T}$ & $\mathrm{ACA}$ & $\mathrm{N}$ & LOW \\
\hline rs7591996 & GRM4 & primary & Per allele & Osteo & 2 & Mixed & $1.28[1.02,1.61]$ & 53 & 0.03 & 1109 & 3507 & $\mathrm{~A} / \mathrm{C}$ & $\mathrm{ACA}$ & $\mathrm{N}$ & LOW \\
\hline deletion & GSTM1 & sensitivity & Recessive & Bone tumor & 3 & Asian & $1.69[1.02,2.81]$ & 66 & 0.04 & 315 & 578 & $\begin{array}{c}\text { non- } \\
\text { null/ } \\
\text { null }\end{array}$ & $\mathrm{BCA}$ & $\mathrm{N}$ & LOW \\
\hline
\end{tabular}

OR [95\%CI]: Summary Odds Ratio [95\% Confidence Interval]; Ref: reference allele; Alt: alternative allele; Venice criteria: A (high), B (moderate), C (weak) credibility for three parameters (amount of evidence, heterogeneity and bias); FPRP: false positive report probability at a prior probability of 10E-3; Y: noteworthy association (FPRP cut-off value 0.2 ), N: non noteworthy association; Level of evidence: overall level of summary evidence according to the Venice criteria and FPRP.

GMR4 (glutamate receptor metabotropic 4), which were part of our meta-analysis and ADAMTS protein family, as ADAM Metallopeptidase with Thrombospondin Type 1 Motif 17 . Of note, most statistically significant associations based on single studies did not have a statistically significant eQTL effect.

\section{Network and pathway analysis findings}

Using the 36 genes whose SNPs were significantly associated with sarcoma risk (including data from both meta-analysis and single studies) and were also characterized by a significant eQTL effect, we found that the corresponding protein products interact with each other beyond chance (observed edges: 120; expected edges: 12; PPI enrichment P-value <10E-20), with an average node degree equal to 6.7 (see Figure 2). Such enrichment indicates that the input molecules - as a whole group - are at least partially biologically connected. This high connectivity prompted us to conduct pathway analysis, which showed that the identified network is significantly enriched in DNA repair proteins, as shown in Table 4.

In particular, many sarcoma risk genes appear to be involved in all main DNA repair pathways, including single strand break repair pathways (base excision repair [BER], nucleotide excision repair [NER], mismatch repair $[\mathrm{MMR}]$ ) and double strand repair pathways (non homologous end joining [NHEJ], homologous recombination $[\mathrm{HR}]$ ).

\section{DISCUSSION}

We described the findings of the first field synopsis and meta-analysis dedicated to the relationship between germline DNA variation and risk of developing bone and soft tissue sarcomas, which is based on genotyping data from 90 studies enrolling almost 48,000 people with a control-to-case ratio equal to 2 . The resulting knowledgebase will be hosted by our cancer-dedicated website (at www.mmmp.org) [100] as a freely available online data repository that will be annually updated.

Overall, our findings support the hypothesis that genetic polymorphism does contribute to sarcoma susceptibility. This is exemplified by the population attributable risk ( $\mathrm{PAR}=37.2 \%$ ) calculated for three SNPs associated with the risk of sarcoma at a high level of evidence (rs11599754 of ZNF365/EGR2, rs231775 of CTLA4, and rs454006 of PRKCG), which indicates that more than one third of sarcoma cases would not occur in a hypothetical population where these three risk variants were absent. This remarkable influence of just three SNPs is linked not only to the high frequency of the risk alleles but also to the interesting fact that the risk, defined as odds ratio, associated with single variants ranged between 1.35 and 1.48 , which are values higher than those usually observed for other malignancies such as breast [101], colorectal [102], and gastric carcinomas [103], which generally include odds ratios between 1.10 and 1.30. Considering that the mean risk among variants significantly associated with sarcoma predisposition was even higher (approximately 1.70, see Table 2), one might speculate that germline DNA variation is especially important in the determinism of the susceptibility to this family of tumors.

Overall, the quality of the available data, which was thoroughly assessed by means of both Venice criteria and false positive report probability (FPRP), was satisfactory considering that the statistically significant evidence on 47 of 55 variants for which a meta-analysis was feasible was classified as high to moderate level of quality with 10 SNPs considered adequate according to the FPRP (Table 2). A statistically significant association was also demonstrated for additional 906 SNPs, for which only a single data source was available, which pinpoints the urgent need for replication studies in order to validate or refute these findings.

Conventional meta-analysis of single variants led us to identify 55 SNPs significantly associated with sarcoma risk (Table 2), and additional 53 SNPs were reported 
Table 3: Statistically significant associations based on single studies (P-value threshold E-06)

\begin{tabular}{|c|c|c|c|c|c|c|c|c|c|c|}
\hline Reference & Cancer type & Genes & SNP ID & $\begin{array}{c}\text { Ref/ } \\
\text { Alt }\end{array}$ & Chr & OR [95\%CI] & P-value & location & eQTL & $\begin{array}{l}\text { eQTL P-value } \\
\text { skeletal muscle }\end{array}$ \\
\hline Postel-Vinay S. [10] & Ewing's & $\begin{array}{l}\text { C1orf127, } \\
\text { TARDBP }\end{array}$ & rs9430161 & $\mathrm{T} / \mathrm{G}$ & 1 & $2.20[1.80,2.70]$ & $1.40 \mathrm{E}-20$ & intergene & & \\
\hline Postel-Vinay S. [10] & Ewing's & C1orf127 & rs 2003046 & $\mathrm{~A} / \mathrm{C}$ & 1 & $1.80[1.50,2.20]$ & $1.30 \mathrm{E}-14$ & intron & & \\
\hline Postel-Vinay S. [10] & Ewing's & C1orf127 & rs 11576658 & $\mathrm{~T} / \mathrm{C}$ & 1 & $1.80[1.40,2.30]$ & $9.40 \mathrm{E}-11$ & intron & & \\
\hline Postel-Vinay S. [10] & Ewing's & SRP14-AS1 & rs 4924410 & $\mathrm{C} / \mathrm{A}$ & 15 & $1.50[1.30,1.70]$ & $6.60 \mathrm{E}-09$ & intron & $\begin{array}{c}\text { RP11- } \\
521 \mathrm{C} 20.2\end{array}$ & $1.60 \mathrm{E}-07$ \\
\hline Grünewald TG. [29] & Ewing's & ADO, EGR2 & rs10995305 & $\mathrm{G} / \mathrm{A}$ & 10 & $1.59[1.26,2.00]$ & $4.38 \mathrm{E}-07$ & intergene & $\mathrm{ADO}$ & $1.40 \mathrm{E}-16$ \\
\hline Zhao J. [97] & Osteo & ARHGAP35 & rs 1052667 & $\mathrm{C} / \mathrm{T}$ & 19 & $2.25[1.64,3.09]$ & $4.43 \mathrm{E}-07$ & utr 3 prime & ARHGAP35 & Other tissue \\
\hline Grünewald TG. [29] & Ewing's & ADO, EGR2 & rs 224290 & $\mathrm{G} / \mathrm{C}$ & 10 & $0.55[0.43,0.70]$ & $7.80 \mathrm{E}-07$ & intergene & $\mathrm{ADO}$ & $7.50 \mathrm{E}-14$ \\
\hline Grünewald TG. [29] & Ewing's & ADO, EGR2 & rs224291 & $\mathrm{G} / \mathrm{A}$ & 10 & $0.55[0.43,0.70]$ & $7.80 \mathrm{E}-07$ & intergene & $\mathrm{ADO}$ & $7.20 \mathrm{E}-14$ \\
\hline Grünewald TG. [29] & Ewing's & ADO, EGR2 & rs224296 & $\mathrm{C} / \mathrm{T}$ & 10 & $0.55[0.43,0.70]$ & $7.80 \mathrm{E}-07$ & intergene & $\mathrm{ADO}$ & $2.90 \mathrm{E}-14$ \\
\hline Grünewald TG. [29] & Ewing's & ADO, EGR2 & rs224297 & $\mathrm{T} / \mathrm{C}$ & 10 & $0.55[0.43,0.70]$ & $7.80 \mathrm{E}-07$ & intergene & $\mathrm{ADO}$ & $2.80 \mathrm{E}-14$ \\
\hline Grünewald TG. [29] & Ewing's & ADO, EGR2 & rs224298 & $\mathrm{G} / \mathrm{A}$ & 10 & $0.55[0.43,0.70]$ & $7.80 \mathrm{E}-07$ & intergene & $\mathrm{ADO}$ & $2.90 \mathrm{E}-14$ \\
\hline Grünewald TG. [29] & Ewing's & ADO, EGR2 & rs224294 & $\mathrm{C} / \mathrm{T}$ & 10 & $0.54[0.43,0.69]$ & $1.01 \mathrm{E}-06$ & intergene & $\mathrm{ADO}$ & $5.60 \mathrm{E}-14$ \\
\hline Grünewald TG. [29] & Ewing's & ADO, EGR2 & rs224293 & $\mathrm{G} / \mathrm{A}$ & 10 & $0.55[0.44,0.71]$ & $1.02 \mathrm{E}-06$ & intergene & $\mathrm{ADO}$ & $7.20 \mathrm{E}-14$ \\
\hline Grünewald TG. [29] & Ewing's & EGR2, ADO & rs 1848796 & $\mathrm{C} / \mathrm{T}$ & 10 & $1.80[1.42,2.29]$ & $1.08 \mathrm{E}-06$ & intergene & $\mathrm{ADO}$ & $2.90 \mathrm{E}-14$ \\
\hline Grünewald TG. [29] & Ewing's & ADO, EGR2 & rs224282 & $\mathrm{A} / \mathrm{G}$ & 10 & $0.55[0.44,0.71]$ & $1.08 \mathrm{E}-06$ & intergene & $\mathrm{ADO}$ & $7.20 \mathrm{E}-14$ \\
\hline Savage SA. [11] & Osteo & ADAMTS17 & rs2086452 & $\mathrm{T} / \mathrm{C}$ & 15 & $1.35[1.19,1.52]$ & $1.12 \mathrm{E}-06$ & intron & & \\
\hline Grünewald TG. [29] & Ewing's & EGR2 & rs648746 & $\mathrm{G} / \mathrm{T}$ & 10 & $0.56[0.44,0.71]$ & $1.21 \mathrm{E}-06$ & upstream & $\mathrm{ADO}$ & $5.10 \mathrm{E}-15$ \\
\hline Grünewald TG. [29] & Ewing's & EGR2 & rs648748 & $\mathrm{G} / \mathrm{A}$ & 10 & $0.56[0.44,0.71]$ & $1.21 \mathrm{E}-06$ & upstream & $\mathrm{ADO}$ & $5.10 \mathrm{E}-15$ \\
\hline Grünewald TG. [29] & Ewing's & EGR2 & rs7076924 & $\mathrm{A} / \mathrm{G}$ & 10 & $1.79[1.41,2.28]$ & $1.21 \mathrm{E}-06$ & upstream & $\mathrm{ADO}$ & $5.50 \mathrm{E}-15$ \\
\hline Grünewald TG. [29] & Ewing's & EGR2 & rs224277 & $\mathrm{T} / \mathrm{C}$ & 10 & $0.56[0.44,0.71]$ & $1.40 \mathrm{E}-06$ & upstream & $\mathrm{ADO}$ & $3.30 \mathrm{E}-15$ \\
\hline Grünewald TG. [29] & Ewing's & ADO, EGR2 & rs224289 & $\mathrm{T} / \mathrm{C}$ & 10 & $0.56[0.44,0.71]$ & $1.42 \mathrm{E}-06$ & intergene & $\mathrm{ADO}$ & $7.20 \mathrm{E}-14$ \\
\hline Grünewald TG. [29] & Ewing's & ADO, EGR2 & rs 7096645 & $\mathrm{G} / \mathrm{T}$ & 10 & $1.78[1.40,2.27]$ & $1.54 \mathrm{E}-06$ & intergene & $\mathrm{ADO}$ & $8.60 \mathrm{E}-14$ \\
\hline Grünewald TG. [29] & Ewing's & $\begin{array}{c}\text { LOC107984012, } \\
\text { NRBF2 }\end{array}$ & rs10740101 & $\mathrm{A} / \mathrm{G}$ & 10 & $2.07[1.55,2.76]$ & 2.29E-06 & intergene & $\mathrm{ADO}$ & $4.90 \mathrm{E}-10$ \\
\hline Grünewald TG. [29] & Ewing's & $\begin{array}{c}\text { EGR2, } \\
\text { LOC107984012 }\end{array}$ & rs7079482 & $\mathrm{C} / \mathrm{T}$ & 10 & $2.06[1.54,2.76]$ & 2.69E-06 & intergene & $\mathrm{ADO}$ & $1.70 \mathrm{E}-10$ \\
\hline Grünewald TG. [29] & Ewing's & $\begin{array}{c}\text { EGR2, } \\
\text { LOC107984012 }\end{array}$ & rs1115705 & $\mathrm{T} / \mathrm{C}$ & 10 & $2.07[1.55,2.77]$ & $2.73 \mathrm{E}-06$ & intergene & $\mathrm{ADO}$ & $9.40 \mathrm{E}-11$ \\
\hline Grünewald TG. [29] & Ewing's & $\begin{array}{c}\text { EGR2, } \\
\text { LOC107984012 }\end{array}$ & rs983319 & $\mathrm{A} / \mathrm{T}$ & 10 & $2.07[1.55,2.77]$ & 2.99E-06 & intergene & $\mathrm{ADO}$ & $4.10 \mathrm{E}-10$ \\
\hline Grünewald TG. [29] & Ewing's & $\begin{array}{c}\text { EGR2, } \\
\text { LOC107984012 }\end{array}$ & rs1571918 & $\mathrm{A} / \mathrm{G}$ & 10 & $2.05[1.54,2.74]$ & $3.44 \mathrm{E}-06$ & intergene & $\mathrm{ADO}$ & $2.80 \mathrm{E}-10$ \\
\hline Grünewald TG. [29] & Ewing's & $\begin{array}{c}\text { EGR2, } \\
\text { LOC107984012 }\end{array}$ & rs1888968 & $\mathrm{C} / \mathrm{T}$ & 10 & $2.05[1.54,2.74]$ & $3.44 \mathrm{E}-06$ & intergene & $\mathrm{ADO}$ & $1.90 \mathrm{E}-10$ \\
\hline Grünewald TG. [29] & Ewing's & $\begin{array}{c}\text { EGR2, } \\
\text { LOC107984012 }\end{array}$ & rs1912369 & $\mathrm{G} / \mathrm{A}$ & 10 & $2.05[1.54,2.74]$ & $3.44 \mathrm{E}-06$ & intergene & $\mathrm{ADO}$ & $3.50 \mathrm{E}-10$ \\
\hline Grünewald TG. [29] & Ewing's & $\begin{array}{c}\text { EGR2, } \\
\text { LOC107984012 }\end{array}$ & rs 4147153 & $\mathrm{~A} / \mathrm{G}$ & 10 & $2.05[1.54,2.74]$ & $3.44 \mathrm{E}-06$ & intergene & $\mathrm{ADO}$ & $3.50 \mathrm{E}-10$ \\
\hline Grünewald TG. [29] & Ewing's & $\begin{array}{c}\text { EGR2, } \\
\text { LOC107984012 }\end{array}$ & rs4237316 & $\mathrm{C} / \mathrm{T}$ & 10 & $2.05[1.54,2.74]$ & $3.44 \mathrm{E}-06$ & intergene & $\mathrm{ADO}$ & $1.90 \mathrm{E}-10$ \\
\hline Grünewald TG. [29] & Ewing's & $\begin{array}{c}\text { EGR2, } \\
\text { LOC107984012 }\end{array}$ & rs4746746 & $\mathrm{C} / \mathrm{T}$ & 10 & $2.05[1.54,2.74]$ & $3.44 \mathrm{E}-06$ & intergene & $\mathrm{ADO}$ & $7.20 \mathrm{E}-10$ \\
\hline Grünewald TG. [29] & Ewing's & $\begin{array}{c}\text { EGR2, } \\
\text { LOC107984012 }\end{array}$ & rs6479854 & $\mathrm{C} / \mathrm{T}$ & 10 & $2.05[1.54,2.74]$ & $3.44 \mathrm{E}-06$ & intergene & $\mathrm{ADO}$ & $1.50 \mathrm{E}-10$ \\
\hline Grünewald TG. [29] & Ewing's & $\begin{array}{c}\text { EGR2, } \\
\text { LOC107984012 }\end{array}$ & rs7100213 & $\mathrm{T} / \mathrm{C}$ & 10 & $2.05[1.54,2.74]$ & $3.44 \mathrm{E}-06$ & intergene & $\mathrm{ADO}$ & $2.10 \mathrm{E}-10$ \\
\hline Grünewald TG. [29] & Ewing's & $\begin{array}{c}\text { EGR2, } \\
\text { LOC107984012 }\end{array}$ & rs4746745 & $\mathrm{T} / \mathrm{C}$ & 10 & $2.03[1.52,2.72]$ & $3.48 \mathrm{E}-06$ & intergene & $\mathrm{ADO}$ & $5.90 \mathrm{E}-11$ \\
\hline Grünewald TG. [29] & Ewing's & ADO, EGR2 & rs224301 & $\mathrm{G} / \mathrm{A}$ & 10 & $0.60[0.47,0.76]$ & 3.67E-06 & intergene & $\mathrm{ADO}$ & $1.20 \mathrm{E}-10$ \\
\hline Grünewald TG. [29] & Ewing's & ADO, EGR2 & rs 224302 & $\mathrm{G} / \mathrm{A}$ & 10 & $0.60[0.47,0.76]$ & 3.67E-06 & intergene & $\mathrm{ADO}$ & $3.70 \mathrm{E}-10$ \\
\hline Grünewald TG. [29] & Ewing's & ADO, EGR2 & rs 10822056 & $\mathrm{C} / \mathrm{T}$ & 10 & $1.65[1.31,2.09]$ & $3.70 \mathrm{E}-06$ & intergene & $\mathrm{ADO}$ & $3.00 \mathrm{E}-13$ \\
\hline Grünewald TG. [29] & Ewing's & ADO, EGR2 & rs224295 & $\mathrm{A} / \mathrm{C}$ & 10 & $0.60[0.48,0.76]$ & $4.80 \mathrm{E}-06$ & intergene & $\mathrm{ADO}$ & $\begin{array}{l}1.50 \mathrm{E}-10 \\
\text { (continued) }\end{array}$ \\
\hline
\end{tabular}




\begin{tabular}{|c|c|c|c|c|c|c|c|c|c|c|}
\hline Reference & Cancer type & Genes & SNP ID & $\begin{array}{c}\text { Ref/ } \\
\text { Alt }\end{array}$ & Chr & OR $[95 \% \mathrm{CI}]$ & P-value & location & eQTL & $\begin{array}{l}\text { eQTL P-value } \\
\text { skeletal muscle }\end{array}$ \\
\hline Grünewald TG. [29] & Ewing's & ADO, EGR2 & rs224299 & $\mathrm{T} / \mathrm{C}$ & 10 & $0.60[0.48,0.76]$ & $4.80 \mathrm{E}-06$ & intergene & $\mathrm{ADO}$ & $1.50 \mathrm{E}-10$ \\
\hline Savage SA. [11] & Osteo & $\begin{array}{l}\text { LOC } 105373401 \\
\text { LOC } 105373402\end{array}$ & rs 13403411 & $\mathrm{C} / \mathrm{T}$ & 2 & $1.30[1.16,1.46]$ & $5.20 \mathrm{E}-06$ & intergene & & \\
\hline Grünewald TG. [29] & Ewing's & $\begin{array}{c}\text { EGR2, } \\
\text { LOC107984012 }\end{array}$ & rs1509952 & $\mathrm{C} / \mathrm{T}$ & 10 & $2.06[1.54,2.76]$ & $5.28 \mathrm{E}-06$ & intergene & $\mathrm{ADO}$ & $3.50 \mathrm{E}-10$ \\
\hline Grünewald TG. [29] & Ewing's & LOC107984012 & rs10740095 & $\mathrm{T} / \mathrm{C}$ & 10 & $2.03[1.52,2.72]$ & $5.50 \mathrm{E}-06$ & intron & $\mathrm{ADO}$ & $4.20 \mathrm{E}-11$ \\
\hline Grünewald TG. [29] & Ewing's & LOC107984012 & rs925307 & $\mathrm{T} / \mathrm{C}$ & 10 & $2.03[1.52,2.72]$ & $5.50 \mathrm{E}-06$ & intron & $\mathrm{ADO}$ & $6.00 \mathrm{E}-11$ \\
\hline Grünewald TG. [29] & Ewing's & $\begin{array}{c}\text { EGR2, } \\
\text { LOC107984012 }\end{array}$ & rs7073383 & $\mathrm{A} / \mathrm{G}$ & 10 & $2.01[1.50,2.69]$ & $5.98 \mathrm{E}-06$ & intergene & $\mathrm{ADO}$ & $1.60 \mathrm{E}-10$ \\
\hline Grünewald TG. [29] & Ewing's & $\begin{array}{c}\text { EGR2, } \\
\text { LOC107984012 }\end{array}$ & rs 10733780 & $\mathrm{G} / \mathrm{T}$ & 10 & $2.01[1.50,2.69]$ & $6.90 \mathrm{E}-06$ & intergene & $\mathrm{ADO}$ & $2.90 \mathrm{E}-10$ \\
\hline Grünewald TG. [29] & Ewing's & LOC107984012 & rs7071512 & $\mathrm{T} / \mathrm{C}$ & 10 & $2.01[1.50,2.69]$ & $6.90 \mathrm{E}-06$ & intron & $\mathrm{ADO}$ & $4.20 \mathrm{E}-11$ \\
\hline Savage SA. [11] & Osteo & $\begin{array}{l}\text { FAM208B, } \\
\text { GDI2 }\end{array}$ & rs2797501 & $\mathrm{A} / \mathrm{G}$ & 10 & $0.62[0.51,0.77]$ & $7.88 \mathrm{E}-06$ & $\begin{array}{c}\text { missense, } \\
\text { downstream }\end{array}$ & & \\
\hline Savage SA. [11] & Osteo & $\begin{array}{c}\text { DLEU1, } \\
\text { LOC107984568 }\end{array}$ & rs573666 & $\mathrm{G} / \mathrm{A}$ & 13 & $0.77[0.68,0.86]$ & $8.59 \mathrm{E}-06$ & intergene & EBPL & Other tissue \\
\hline Grünewald TG. [29] & Ewing's & $\begin{array}{c}\text { EGR2, } \\
\text { LOC107984012 }\end{array}$ & rs10740097 & $\mathrm{C} / \mathrm{T}$ & 10 & $2.03[1.51,2.72]$ & $9.03 \mathrm{E}-06$ & intergene & $\mathrm{ADO}$ & $1.20 \mathrm{E}-10$ \\
\hline Grünewald TG. [29] & Ewing's & LOC107984012 & rs6479848 & $\mathrm{T} / \mathrm{C}$ & 10 & $2.01[1.50,2.69]$ & $9.16 \mathrm{E}-06$ & intron & $\mathrm{ADO}$ & $2.70 \mathrm{E}-11$ \\
\hline Grünewald TG. [29] & Ewing's & $\begin{array}{c}\text { ZNF365, ADO, } \\
\text { EGR2 }\end{array}$ & rs224079 & $\mathrm{C} / \mathrm{T}$ & 10 & $1.58[1.25,2.01]$ & $9.24 \mathrm{E}-06$ & intergene & $\mathrm{ADO}$ & $5.00 \mathrm{E}-22$ \\
\hline Grünewald TG. [29] & Ewing's & LOC107984012 & rs965128 & $\mathrm{C} / \mathrm{T}$ & 10 & $1.99[1.49,2.66]$ & $9.48 \mathrm{E}-06$ & intron & $\mathrm{ADO}$ & $3.10 \mathrm{E}-11$ \\
\hline
\end{tabular}

OR [95\%CI]: Odds Ratio [95\% Confidence Interval]; Ref: reference allele; Alt: alternative allele; eQTL: expression quantitative trait locus.

in single studies (Table 3): these variants are linked to a variety of genes whose protein products are involved in several cell activities. Therefore, we tried to provide readers with a preliminary interpretation of these findings from the functional biology viewpoint. Using modern SNP-to-gene and gene-to-function approaches such as integrative analysis of genetic variation with expression quantitative trait locus (eQTL) data [9] and respectively pathway/network analysis [8], we hypothesize that germline variation of the DNA repair machinery might be of special relevance for the development of this type of cancer (Figure 2). This finding - which has been very recently confirmed in patients with Ewing's sarcoma [104] - is in line with the complex gene and chromosome abnormalities that characterized some sarcoma histologies, as well as with the epidemiological observation that people accidentally [105] or therapeutically [106] exposed to ionizing radiations and thus prone to develop DNA damage are at higher risk of different types of sarcomas. In this regard, it is interesting to note that peripheral blood mononuclear cells of patients diagnosed with sarcomas show a higher sensitivity to mutagens in vitro as compared to controls [107], which supports the hypothesis that the genetic background can make the difference on an individual basis in terms of response to environmental carcinogens potentially involved in sarcomagenesis.

Finally, also somatic DNA alterations appear to confer a defective DNA repair capability to some sarcoma types such as Ewing's sarcoma [108], and thus the combinatory study of germline and somatic DNA variations characterizing sarcomas might lead to better understand the cascade of molecular events underlying sarcomagenesis, as recently proposed for the EWSRI-FLII fusion gene and the SNPs near EGR2 in Ewing's sarcoma patients [29].

Overall, these converging data suggest that more investigation aimed to fully elucidate whether the germline individual capacity of repairing genomic damage can actually affect the predisposition to a complex and heterogeneous trait such as sarcomas might be particularly fruitful.

In our work we also confirmed the association between sarcoma risk and variants of single genes, such as ZNF365, ADO, EGR2, CTLA4, TP53, CD86, NUDT6, MDM2, ERCC5 and ADAMTS6 just to mention the top ten by statistical significance. Many of these genes are not known to be involved in DNA repair and thus the relationship between these single gene findings and network/pathway analysis might appear of unclear interpretation and doubtful importance. However, we must remember that current evidence (and thus our analysis) is based on 88 candidate gene studies and only two GWAS: therefore, more extensive investigation is needed on the variation of pathways for which data on single genes are currently available. In this regard, our meta-analysis data can be utilized to inform future studies on candidate pathways whose genetic variation could affect sarcoma susceptibility. 
This systematic review also underscores the main limitation of the evidence on the genetic susceptibility of sarcomas. In fact, most of current information is driven by data from studies investigating bone tumors (78 of 90 , $86.6 \%$ ). Studies focusing on soft tissue sarcomas are thus eagerly awaited, the formation of international consortia being advocated in order to overcome the hurdle of disease rarity. Hopefully, technological improvements in direct DNA sequencing such as next generation sequencing (NGS) methods will further accelerate the discovery pace in this field of investigation, as recently reported [104].
Nevertheless, we also recognize some limitations of this synopsis: data from different tumor types and population ethnicity were pooled together to find associations despite the diversity of sarcoma histologies, leading to high level of between-study heterogeneity. To overcome to this limitation we performed subgroup and sensitivity analysis whenever possible. Moreover, despite our efforts to avoid the issue of overlapping series, it is always possible that partial overlaps between multiple series published by the same research groups that cannot be detected by full text reading did remain included in pooled analyses: however, we believe that the influence

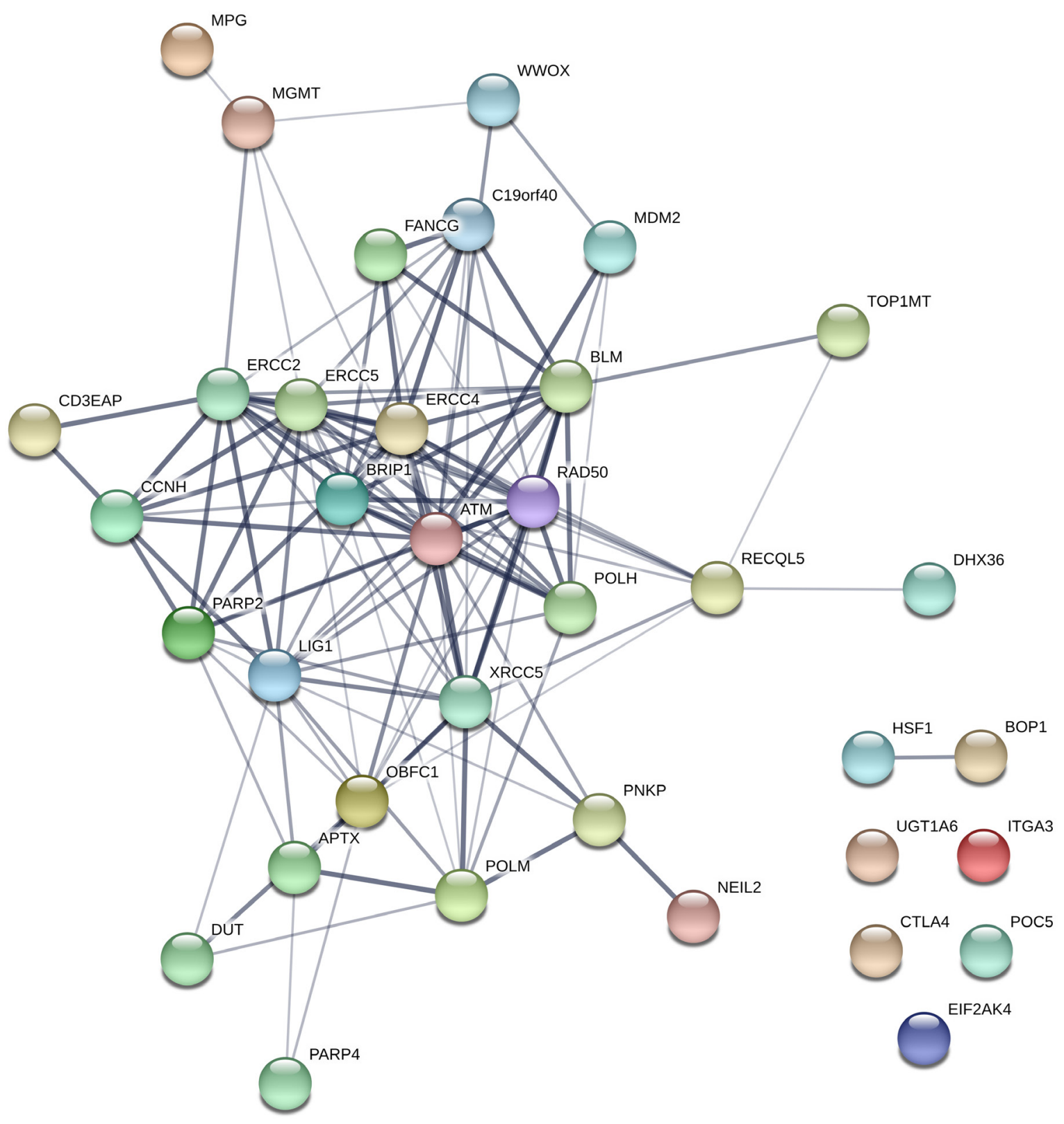

Figure 2: Network analysis of proteins encoded by genes whose variants associated with sarcoma risk and characterized by an expression quantitative trait locus effect (eQTL). The figure illustrates the high degree of connectivity of these proteins, which result to be enriched in DNA repair pathway components. 
Table 4: Pathway analysis main findings: gene set enrichment analysis based on 36 sarcoma risk genes. Enrichments with at least ten overlapping genes are shown

\begin{tabular}{|c|c|c|c|c|}
\hline Pathway & Overlap & FDR & Genes & Database \\
\hline $\begin{array}{l}\text { Base excision repair } \\
(\mathrm{BER})\end{array}$ & $11 / 139$ & 0.002374441 & $\begin{array}{l}\text { BLM;RAD50; PARP4; } \\
\text { RECQL5; LIG1; MPG; } \\
\text { PARP2; ERCC4; PNKP; } \\
\text { FANCG; POLH }\end{array}$ & GO biol process \\
\hline $\begin{array}{l}\text { DNA 3' } \\
\text { dephosphorylation } \\
\text { involved in DNA repair }\end{array}$ & $10 / 120$ & 0.002376199 & $\begin{array}{l}\text { BLM; RAD50; PARP4; } \\
\text { RECQL5; LIG1; PARP2; } \\
\text { ERCC4; PNKP; FANCG; } \\
\text { POLH }\end{array}$ & GO biol process \\
\hline $\begin{array}{l}\text { DNA dealkylation } \\
\text { involved in DNA repair }\end{array}$ & $12 / 128$ & 0.000983329 & $\begin{array}{l}\text { BLM; RAD50; PARP4; } \\
\text { RECQL5; LIG1; MPG; } \\
\text { MGMT; PARP2; ERCC4; } \\
\text { PNKP; FANCG; POLH }\end{array}$ & GO biol process \\
\hline $\begin{array}{l}\text { DNA ligation involved in } \\
\text { DNA repair }\end{array}$ & $11 / 132$ & 0.002374441 & $\begin{array}{l}\text { BLM; RAD50; PARP4; } \\
\text { RECQL5; LIG1; MGMT; } \\
\text { PARP2; ERCC4; PNKP; } \\
\text { FANCG; POLH }\end{array}$ & GO biol process \\
\hline DNA repair & $18 / 285$ & $8.22494 \mathrm{E}-05$ & $\begin{array}{l}\text { BLM; LIG1; CCNH; } \\
\text { XRCC5; PARP2; MGMT; } \\
\text { MPG; POLM; PNKP; } \\
\text { FANCG; BRIP1; RAD50; } \\
\text { NEIL2; ERCC4; ERCC2; } \\
\text { ATM; ERCC5; POLH }\end{array}$ & Reactome \\
\hline $\begin{array}{l}\text { DNA synthesis involved } \\
\text { in DNA repair }\end{array}$ & $12 / 142$ & 0.001514863 & $\begin{array}{l}\text { BLM; BRIP1; RAD50; } \\
\text { PARP4; RECQL5; LIG1; } \\
\text { PARP2; ERCC4; PNKP; } \\
\text { ATM; FANCG; POLH }\end{array}$ & GO biol process \\
\hline $\begin{array}{l}\text { Double-strand break } \\
\text { repair (DSBR) }\end{array}$ & $12 / 164$ & 0.002374441 & $\begin{array}{l}\text { BLM; BRIP1; RAD50; } \\
\text { PARP4; RECQL5; } \\
\text { LIG1; XRCC5; PARP2; } \\
\text { ERCC4; PNKP; FANCG; } \\
\text { POLH }\end{array}$ & GO biol process \\
\hline Mismatch repair (MMR) & $10 / 140$ & 0.005835867 & $\begin{array}{l}\text { BLM; RAD50; PARP4; } \\
\text { RECQL5; LIG1; PARP2; } \\
\text { ERCC4; PNKP; FANCG; } \\
\text { POLH }\end{array}$ & GO biol process \\
\hline $\begin{array}{l}\text { Mitochondrial DNA } \\
\text { repair }\end{array}$ & $10 / 123$ & 0.002552586 & $\begin{array}{l}\text { BLM; RAD50; PARP4; } \\
\text { RECQL5; LIG1; PARP2; } \\
\text { ERCC4; PNKP; FANCG; } \\
\text { POLH }\end{array}$ & GO biol process \\
\hline $\begin{array}{l}\text { Non homologous end } \\
\text { joining (NHEJ) }\end{array}$ & $10 / 120$ & 0.002376199 & $\begin{array}{l}\text { BLM; RAD50; PARP4; } \\
\text { RECQL5; LIG1; PARP2; } \\
\text { ERCC4; PNKP; FANCG; } \\
\text { POLH }\end{array}$ & GO biol process \\
\hline $\begin{array}{l}\text { Nucleotide excision repair } \\
\text { (NER) }\end{array}$ & $11 / 138$ & 0.002374441 & $\begin{array}{l}\text { BLM; RAD50; PARP4; } \\
\text { RECQL5; LIG1; PARP2; } \\
\text { ERCC4; PNKP; ERCC2; } \\
\text { FANCG; POLH }\end{array}$ & GO biol process \\
\hline
\end{tabular}




\begin{tabular}{|c|c|c|c|c|}
\hline Pathway & Overlap & FDR & Genes & Database \\
\hline $\begin{array}{l}\text { Nucleotide } \\
\text { phosphorylation involved } \\
\text { in DNA repair }\end{array}$ & $10 / 120$ & 0.002376199 & $\begin{array}{l}\text { BLM; RAD50; PARP4; } \\
\text { RECQL5; LIG1; PARP2; } \\
\text { ERCC4; PNKP; FANCG; } \\
\text { POLH }\end{array}$ & GO biol process \\
\hline $\begin{array}{l}\text { Homologous } \\
\text { recombination (HR) }\end{array}$ & $10 / 132$ & 0.00369711 & $\begin{array}{l}\text { BLM; RAD50; PARP4; } \\
\text { RECQL5; LIG1; PARP2; } \\
\text { ERCC4; PNKP; FANCG; } \\
\text { POLH }\end{array}$ & GO biol process \\
\hline $\begin{array}{l}\text { Single strand break repair } \\
\text { (SSBR) }\end{array}$ & $11 / 124$ & 0.001805921 & $\begin{array}{l}\text { BLM; RAD50; PARP4; } \\
\text { RECQL5; LIG1; PARP2; } \\
\text { ERCC4; PNKP; APTX; } \\
\text { FANCG; POLH }\end{array}$ & GO biol process \\
\hline $\begin{array}{l}\text { UV-damage excision } \\
\text { repair }\end{array}$ & $11 / 158$ & 0.003533915 & $\begin{array}{l}\text { BLM; RAD50; PARP4; } \\
\text { RECQL5; LIG1; } \\
\text { PARP2; ERCC4; PNKP; } \\
\text { EIF2AK4; FANCG; } \\
\text { POLH }\end{array}$ & GO biol process \\
\hline XPC complex (NER) & $15 / 160$ & 8.19637E-06 & $\begin{array}{l}\text { WWOX; CCNH; } \\
\text { XRCC5; MGMT; } \\
\text { CD3EAP; FANCG; } \\
\text { POC5; ERCC4; ERCC2; } \\
\text { MDM2; OBFC1; ATM; } \\
\text { ERCC5; POLH; UGT1A6 }\end{array}$ & Jenesen compartments \\
\hline
\end{tabular}

FDR: false discovery rate.

of this potential residual overlapping on the overall results is reasonably low.

In conclusion, we hope that the creation of the first knowledgebase dedicated to the relationship between germline DNA variation and sarcoma risk can not only represent a valuable reference for investigators involved in sarcoma research but also inform future studies based on the gaps of the current literature.

\section{MATERIALS AND METHODS}

\section{Search strategy, eligibility criteria, quality score assessment and data extraction}

This study followed the principles proposed by the Human Genome Epidemiology Network (HuGeNet) for the systematic review of molecular association studies [109].

We considered eligible all the studies concerning the association between any genetic variant and the predisposition to sarcoma in humans, providing the raw data necessary to calculate risk of developing a sarcoma or the summary data. Exclusion criteria were: virusinduced sarcomas (HHV8 - Kaposi sarcoma); sarcomas secondary to radiation therapy; sarcomas secondary to burns/scars/surgery; associations between mitochondrial
DNA variations and sarcomas; gastrointestinal stromal tumors (GIST).

Database search of original articles analyzing the association between any genetic variant and susceptibility to sarcoma was conducted independently by two investigators though the following database: MEDLINE (via the PubMed gateway); The Cochrane Library; Scopus; Web of Science. The search included the following three groups of keywords: 1) sarcoma, solitary fibrous tumor, chordoma, tenosynovitis, fibromatosis, desmoids, myofibroblastic, myopericytoma, myxoma, Ewing, desmoplastic, PEComa, haemangioendothelioma, lymphangioma, myoepithelioma; 2) risk, sarcomagenesis, tumorigenesis, predisposition, susceptibility; 3) polymorphism, SNP, variant, genome wide association study and its acronym GWAS. Searches were conducted using all combinations of at least one keyword from each group. References from eligible articles were also used to refine the literature search.

The quality of the studies was evaluated according to Newcastle-Ottawa quality assessment scale (NOS) [110]. In brief, the following three parameters were evaluated with a "star system": the selection of the study groups ( 0 to 4 "stars"), the comparability of the groups (0 to 2 "stars"), and the ascertainment of either the exposure or outcome of interest for case-control or cohort studies 
respectively (0 to 3 "stars"). The maximum total score was 9 "stars" and represented the highest quality.

Data were extracted independently by two investigators using a template. Every disagreement was resolved by a third investigator in order to reach consensus. Authors were contacted whenever unreported data were potentially useful to enable the inclusion of the study into the systematic review. The data extracted from eligible studies were: authors, journal, year of publication, region or country where the study was conducted, hospital where the patients were diagnosed, number of patients with sarcoma enrolled and healthy control subjects, period of enrolment, prevalent ethnicity $(>80 \%$, categorized in Caucasian, Asian, African and mixed), subjects age, genetic polymorphisms and allelic frequency in both cases and controls (if no raw data were available, summary data were collected, i.e. odds ratios and confidence intervals), study design (population-based versus hospital-based), statistical methods used, and sarcoma histology.

We considered data published in different articles by the same Author/s with the same (or similar) number of subjects enrolled in the same period of time in the same hospital, to be derived by the same group of patients. In publications with either overlapping cases or controls, the most recent or largest population was chosen.

For analysis purposes, the search was closed in August 2017.

\section{Statistical analysis}

We calculated summary odds ratios (ORs) and their corresponding $95 \%$ confidence intervals $(95 \% \mathrm{CI})$ starting from raw data to measure the strength of association between each polymorphism and sarcoma risk.

Whenever possible, we calculated the pooled ORs assuming 3 different genetic models: per-allele (additive), dominant and recessive. If the included studies reported exclusively per-allele ORs, as in GWAS, we calculated the pooled OR assuming the per-allele (additive) model.

Random effects meta-analysis based on the inverse variance method was used to calculate summary ORs; this model reduces to a fixed effect meta-analysis if betweenstudy heterogeneity is absent. We chose this model for the large between-study heterogeneity usually expected in genetic association studies. A meta-analysis was performed only if at least two independent data sources were available. In case of GWAS, we considered as data source the joint analysis between the discovery and the validation phases. Subgroup analysis by histological subtype (Ewing's sarcoma vs osteosarcoma) was planned if data permitted.

Regarding ethnicity, analyses were divided in 4 groups: African (if the datasets were all African population-based), Asian (if the datasets were all Asian population-based), Caucasian (if the datasets were all Caucasian population-based), and mixed (if the datasets were African, Asian and Caucasian or if the datasets were from mixed ethnicity). In order to test any dominant study driving effect, sensitivity analysis by ethnicity (Asian vs Caucasian/other) was performed in mixed meta-analyses, with more than two datasets, excluding either the Asian study or the Caucasian study from the meta-analysis.

Between-study heterogeneity was formally assessed by the Cochran Q-test and the I-squared statistic, the latter indicating the proportion of the variability in effect estimates linked to true between-study heterogeneity as opposed to within-study sampling error.

All statistical analyses were performed with RevMan 5 (Review Manager computer program, version 5.3; Copenhagen, The Nordic Cochrane Centre, The Cochrane Collaboration, 2014).

\section{Assessment of cumulative evidence}

With the aim to assess the credibility of statistically significant associations based on the results of data metaanalysis, we used the Venice criteria [111]. In brief, we defined credibility levels based on the strength (classified as $\mathrm{A}=$ strong, $\mathrm{B}=$ moderate or $\mathrm{C}=$ weak) of three following parameters: amount of the evidence, replication of the association and protection from bias. We graded the amount of evidence, which approximately depends on the study sample size, based on the sum of cases and controls. Grade A, B or C was assigned to meta-analyses with total sample size $>1000,100-1000$ and $<100$, respectively. Also, the replication of the association was graded considering the amount of between-study heterogeneity. We assigned grade $\mathrm{A}, \mathrm{B}$ or $\mathrm{C}$ to meta-analyses with I-squared $<25 \%, 25-50 \%$ and $>50 \%$, respectively. We graded protection from bias as A if no bias was observed, $\mathrm{B}$ if bias was potentially present or $\mathrm{C}$ if bias was evident. While assessing protection from bias we also considered the magnitude of the association. We assigned a score of $\mathrm{C}$ to an association characterized by a summary $\mathrm{OR}<1.15$ or a summary $\mathrm{OR}>0.87$ if the effect of the polymorphism was protective.

In addition to the Venice criteria, we assessed the noteworthiness of significant findings by calculating the false positive report probability (FPRP) [112], which is defined as the probability of no true association between a genetic variant and disease (null hypothesis) given a statistically significant finding. FPRP is based not only on the observed P-value of the association test but also on the statistical power of the test and on the prior probability that the molecular association is real following a Bayesian approach. We calculated FPRP values for two levels of prior probabilities: at a low prior (10E-3) that would be similar to what is expected for a candidate variant, and at a very low prior (10E-6) that would be similar to what would be expected for a random variant. To classify a significant association as 'noteworthy', we used a FPRP cut-off value of 0.2 . 
Overall, we defined the credibility level of the cumulative evidence as high (Venice criteria A grades only coupled with "noteworthy" finding at FPRP analysis), low (one or more $\mathrm{C}$ grades combined with lack of noteworthiness), or intermediate (for all other combinations).

To estimate the impact of genetic variation on the risk of sarcomas, we calculated the so called population attributable risk (PAR) using the following formula:

$$
\operatorname{Pr}(R R-1) /[1+\operatorname{Pr}(R R-1)] \text {, }
$$

where $\mathrm{Pr}$ is the proportion of control subjects exposed to the allele of interest and the relative risk (RR) was estimated using the summary estimates (i.e. ORs) calculated by the meta-analysis. The joint PAR for combinations of polymorphisms was calculated as follows:

$$
1-\left(\prod_{1 \rightarrow n}[1-\text { PARi }]\right) \text {, }
$$

where PARi corresponds to the individual PAR of the $i$ th polymorphism and $n$ is the number of polymorphisms considered [113].

\section{Network and pathway analysis}

In order to explore the mechanisms underlying the pathogenesis of sarcomas, we utilized network and pathway analysis to test the hypothesis that genes whose variations are associated with sarcoma risk interact with each other possibly within the frame of some specific molecular pathways [8].

To this aim, we first selected SNPs significantly associated with sarcoma risk. In case of SNPs located in intergenic regions we selected the first closest and the second closest genes, not necessarily upstream and downstream of the SNPs of interest.

Since most SNPs are intergenic or intronic and thus no obvious functional effect can be inferred, expression quantitative trait locus (eQTL) analysis was used to identify genes whose expression is affected by DNA variants [114]. The resulting gene list was the input for both network and pathway analysis.

For the former, the STRING web server was employed to study protein-protein interaction (PPI) across the selected genes [115], the confidence score being set $>0.4$. As a measure of across network connectivity STRING provides the average node degree, where degree is the conceptually simplest centrality measure as it measures the number of edges between protein connections attached to a protein; moreover, STRING computes the PPI enrichment P-value, which is significant when input proteins have more interactions among themselves than what would be expected for a random set of proteins of similar size, drawn from the genome.

As regards pathway analysis, the Enrichr web server was utilized to identify in our list over-representation of genes involved in specific pathways described in dedicated databases [116]. Hypergeometric distribution with Fisher's exact test was used to calculate the statistical significance of gene overlapping, followed by correction for multiple hypotheses testing using the false discovery rate [FDR] method.

\section{Declarations}

Ethics approval and consent to participate: Not applicable

Consent for publication: Not applicable

Availability of data and material: All data generated or analysed during this study are included in this published article [and its supplementary information files].

\section{Authors' contributions}

CB, AS, DDB, SR, GS: database search and data extraction; $\mathrm{CC}, \mathrm{CV}$ : data revision, quality score assessment; $\mathrm{CB}$, AS: statistical analysis, assessment of cumulative evidence and manuscript writing; SP, SM: network/pathway analysis, manuscript writing and revision; SGDB, AG, CRR: appraisal of manuscript.

\section{CONFLICTS OF INTEREST}

The authors declare that they have no conflicts of interests.

\section{FUNDING}

University of Padova, BIRD168075, "Germline polymorphisms of candidate genes as predictor of risk and prognosis in patients with cutaneous melanoma and soft tissue sarcoma."

\section{REFERENCES}

1. Taylor BS, Barretina J, Maki RG, Antonescu CR, Singer $\mathrm{S}$, Ladanyi $\mathrm{M}$. Advances in sarcoma genomics and new therapeutic targets. Nature reviews.Cancer. 2011; 11: 541-557.

2. Helman LJ, Meltzer P. Mechanisms of sarcoma development. Nature reviews.Cancer. 2003; 3: 685-694.

3. Farid M, Ngeow J. Sarcomas Associated With Genetic Cancer Predisposition Syndromes: A Review. Oncologist. 2016; 21: 1002-1013.

4. Sobhan MR, Forat Yazdi M, Mazaheri M, Zare Shehneh M, Neamatzadeh H. Association between the DNA Repair Gene XRCC3 rs861539 Polymorphism and Risk of Osteosarcoma: a Systematic Review and Meta-Analysis. Asian Pac J Cancer Prev. 2017; 18: 549-555.

5. Zhang D, Ding Y, Wang Z, Wang Y, Zhao G. Impact of MDM2 gene polymorphism on sarcoma risk. Tumour Biol. 2015; 36: 1791-1795. 
6. Bilbao-Aldaiturriaga N, Askaiturrieta Z, Granado-Tajada I, Goricar K, Dolzan V, Garcia-Miguel P, Garcia de Andoin N, Martin-Guerrero I, Garcia-Orad A, For The Slovenian Osteosarcoma Study Group. A systematic review and meta-analysis of MDM2 polymorphisms in osteosarcoma susceptibility. Pediatric research. 2016; 80: 472-479.

7. Bilbao-Aldaiturriaga N, Patino-Garcia A, Martin-Guerrero I, Garcia-Orad A. Cytotoxic T lymphocyte-associated antigen 4 rs231775 polymorphism and osteosarcoma. Neoplasma. 2017; 64: 299-304.

8. Creixell P, Reimand J, Haider S, Wu G, Shibata T, Vazquez M, Mustonen V, Gonzalez-Perez A, Pearson J, Sander C, Raphael BJ, Marks DS, Ouellette BFF, et al. Pathway and network analysis of cancer genomes. Nat Methods. 2015; 12: 615-621.

9. Kristensen VN, Lingjaerde OC, Russnes HG, Vollan HK, Frigessi A, Borresen-Dale AL. Principles and methods of integrative genomic analyses in cancer. Nature reviews. Cancer. 2014; 14: 299-313.

10. Postel-Vinay S, Veron AS, Tirode F, Pierron G, Reynaud S, Kovar H, Oberlin O, Lapouble E, Ballet S, Lucchesi C, Kontny U, Gonzalez-Neira A, Picci P, et al. Common variants near TARDBP and EGR2 are associated with susceptibility to Ewing sarcoma. Nat Genet. 2012; 44: 323-327.

11. Savage SA, Mirabello L, Wang Z, Gastier-Foster JM, Gorlick R, Khanna C, Flanagan AM, Tirabosco R, Andrulis IL, Wunder JS, Gokgoz N, Patino-Garcia A, Sierrasesumaga L, et al. Genome-wide association study identifies two susceptibility loci for osteosarcoma. Nat Genet. 2013; 45: 799-803.

12. Adiguzel M, Horozoglu C, Kilicoglu O, Ozger H, Acar L, Ergen A. MMP-3 gene polymorphisms and Osteosarcoma. Indian J Exp Biol. 2016; 54: 175-179.

13. Alhopuro P, Ylisaukko-Oja SK, Koskinen WJ, Bono P, Arola J, Jarvinen HJ, Mecklin JP, Atula T, Kontio R, Makitie AA, Suominen S, Leivo I, Vahteristo P, et al. The MDM2 promoter polymorphism SNP309T-->G and the risk of uterine leiomyosarcoma, colorectal cancer, and squamous cell carcinoma of the head and neck. J Med Genet. 2005; 42: 694-698.

14. Almeida PS, Manoel WJ, Reis AA, Silva ER, Martins E, Paiva MV, Fraga AC Jr, Saddi VA. TP53 codon 72 polymorphism in adult soft tissue sarcomas. Genet Mol Res. 2008; 7: 1344-1352.

15. Aoyama T, Nagayama S, Okamoto T, Hosaka T, Nakamata T, Nishijo K, Tsuboyama T, Nakayama T, Nakamura T, Toguchida J. Mutation analyses of the NFAT1 gene in chondrosarcomas and enchondromas. Cancer Lett. 2002; 186: 49-57.

16. Barnette P, Scholl R, Blandford M, Ballard L, Tsodikov A, Magee J, Williams S, Robertson M, Ali-Osman F, Lemons R, Keller C. High-throughput detection of glutathione s-transferase polymorphic alleles in a pediatric cancer population. Cancer Epidemiol Biomark Prevent. 2004; 13: 304-313.

17. Biason $\mathrm{P}$, Hattinger CM, Innocenti F, Talamini R, Alberghini M, Scotlandi K, Zanusso C, Serra M, Toffoli G. Nucleotide excision repair gene variants and association with survival in osteosarcoma patients treated with neoadjuvant chemotherapy. Pharmacogenomics J. 2012; 12: 476-483.

18. Bilbao-Aldaiturriaga N, Gutierrez-Camino A, MartinGuerrero I, Pombar-Gomez M, Zalacain-Diez M, PatinoGarcia A, Lopez-Lopez E, Garcia-Orad A. Polymorphisms in miRNA processing genes and their role in osteosarcoma risk. Pediatr Blood Cancer. 2015; 62: 766-769.

19. Chen Y, Yang Y, Liu S, Zhu S, Jiang H, Ding J. Association between interleukin $8-251 \mathrm{~A} / \mathrm{T}$ and +781 $\mathrm{C} / \mathrm{T}$ polymorphisms and osteosarcoma risk in Chinese population: a case-control study. Tumour Biol. 2016; 37: 6191-6196.

20. Cong Y, Li CJ, Zhao JN, Liu XZ, Shi X. Associations of polymorphisms in the bone morphogenetic protein-2 gene with risk and prognosis of osteosarcoma in a Chinese population. Tumour Biol. 2015; 36: 2059-2064.

21. Cui Y, Zhu JJ, Ma CB, Cui K, Wang F, Ni SH, Zhang ZY. Genetic polymorphisms in MMP 2, 3 and 9 genes and the susceptibility of osteosarcoma in a Chinese Han population. Biomarkers. 2016; 21: 160-163.

22. Cui Y, Zhu JJ, Ma CB, Cui K, Wang F, Ni SH, Zhang ZY. Interleukin 10 gene $-1082 \mathrm{~A} / \mathrm{G}$ polymorphism is associated with osteosarcoma risk and poor outcomes in the Chinese population. Tumour Biol. 2016; 37: 4517-4522.

23. Dong $\mathrm{YZ}$, Huang $\mathrm{YX}, \mathrm{Lu} \mathrm{T}$. Single nucleotide polymorphism in the RECQL5 gene increased osteosarcoma susceptibility in a Chinese Han population. Genet Mol Res. 2015; 14: 1899-1902.

24. DuBois SG, Goldsby R, Segal M, Woo J, Copren K, Kane JP, Pullinger CR, Matthay KK, Witte J, Lessnick SL, Robison LL, Bhatia S, Strong LC. Evaluation of polymorphisms in EWSR1 and risk of Ewing sarcoma: a report from the Childhood Cancer Survivor Study. Pediatr Blood Cancer. 2012; 59: 52-56.

25. Ergen A, Kilicoglu O, Ozger H, Agachan B, Isbir T. Paraoxonase 1192 and 55 polymorphisms in osteosarcoma. Mol Biol Rep. 2011; 38: 4181-4184.

26. Feng D, Yang X, Li S, Liu T, Wu Z, Song Y, Wang J, Gao W, Huang Q, Huang W, Zheng W, Xiao J. Cytotoxic T-lymphocyte antigen-4 genetic variants and risk of Ewing's sarcoma. Genet Test Mol Biomark. 2013; 17: 458-463.

27. Gloudemans T, Pospiech I, Van der Ven LT, Lips CJ, Den Otter W, Sussenbach JS. An avaII restriction fragment length polymorphism in the insulin-like growth factor II gene and the occurrence of smooth muscle tumors. Cancer Res. 1993; 53: 5754-5758.

28. Grochola LF, Vazquez A, Bond EE, Wurl P, Taubert H, Muller TH, Levine AJ, Bond GL. Recent natural selection identifies a genetic variant in a regulatory subunit of protein 
phosphatase $2 \mathrm{~A}$ that associates with altered cancer risk and survival. Clin Cancer Res. 2009; 15: 6301-6308.

29. Grunewald TG, Bernard V, Gilardi-Hebenstreit P, Raynal V, Surdez D, Aynaud MM, Mirabeau O, Cidre-Aranaz F, Tirode F, Zaidi S, Perot G, Jonker AH, Lucchesi C, et al. Chimeric EWSR1-FLI1 regulates the Ewing sarcoma susceptibility gene EGR2 via a GGAA microsatellite. Nat Genet. 2015; 47: 1073-1078.

30. Guo J, Lv HC, Shi RH, Liu WL. Association between XRCC3 Thr241Met polymorphism and risk of osteosarcoma in a Chinese population. Genet Mol Res. 2015; 14: 16484-16490.

31. He J, Wang J, Wang D, Dai S, Yv T, Chen P, Ma R, Diao C, Lv G. Association analysis between genetic variants of MDM2 gene and osteosarcoma susceptibility in Chinese. Endocr J. 2013; 60: 1215-1220.

32. He J, Wang J, Wang D, Dai S, Yv T, Chen P, Ma R, Diao C, Lv G. Association between CTLA-4 genetic polymorphisms and susceptibility to osteosarcoma in Chinese Han population. Endocrine. 2014; 45: 325-330.

33. He M, Wang Z, Zhao J, Chen Y, Wu Y. COL1A1 polymorphism is associated with risks of osteosarcoma susceptibility and death. Tumour Biol. 2014; 35: 1297-1305.

34. He ML, Wu Y, Zhao JM, Wang Z, Chen YB. PIK3CA and AKT gene polymorphisms in susceptibility to osteosarcoma in a Chinese population. Asian Pac J Cancer Prevent. 2013; 14: 5117-5122.

35. He Y, Liang X, Meng C, Shao Z, Gao Y, Wu Q, Liu J, Wang H, Yang S. Genetic polymorphisms of interleukin-1 beta and osteosarcoma risk. Int Orthop. 2014; 38: 1671-1676.

36. Hu GL, Ma G, Ming JH. Impact of common SNPs in VEGF gene on the susceptibility of osteosarcoma. Genet Mol Res. 2015; 14: 14561-14566.

37. Hu YS, Pan Y, Li WH, Zhang Y, Li J, Ma BA. Association between TGFBR1*6A and osteosarcoma: a Chinese casecontrol study. BMC Cancer. 2010; 10: 169-2407-10-169.

38. Hu YS, Pan Y, Li WH, Zhang Y, Li J, Ma BA. Int7G24A variant of transforming growth factor-beta receptor 1 is associated with osteosarcoma susceptibility in a Chinese population. Med Oncol. 2011; 28: 622-625.

39. $\mathrm{Hu} \mathrm{Z}$, Li N, Xie X, Jiang R. The association of MDM2 c. $346 \mathrm{G}>\mathrm{A}$ genetic variant with the risk of osteosarcoma in Chinese. Genet Test Mol Biomark. 2015; 19: 108-111.

40. Ito M, Barys L, O'Reilly T, Young S, Gorbatcheva B, Monahan J, Zumstein-Mecker S, Choong PF, Dickinson I, Crowe P, Hemmings C, Desai J, Thomas DM, et al. Comprehensive mapping of p53 pathway alterations reveals an apparent role for both SNP309 and MDM2 amplification in sarcomagenesis. Clin Cancer Res. 2011; 17: 416-426.

41. Jiang C, Chen H, Shao L, Dong Y. GRM4 gene polymorphism is associated with susceptibility and prognosis of osteosarcoma in a Chinese Han population. Med Oncol. 2014; 31: 50.
42. Kelley MJ, Shi J, Ballew B, Hyland PL, Li WQ, Rotunno M, Alcorta DA, Liebsch NJ, Mitchell J, Bass S, Roberson D, Boland J, Cullen M, et al. Characterization of T gene sequence variants and germline duplications in familial and sporadic chordoma. Hum Genet. 2014; 133: 1289-1297.

43. Koshkina NV, Kleinerman ES, Li G, Zhao CC, Wei Q, Sturgis EM. Exploratory analysis of Fas gene polymorphisms in pediatric osteosarcoma patients. J Pediatr Hematol Oncol. 2007; 29: 815-821.

44. Le Morvan V, Longy M, Bonaiti-Pellie C, Bui B, Houede N, Coindre JM, Robert J, Pourquier P. Genetic polymorphisms of the XPG and XPD nucleotide excision repair genes in sarcoma patients. Int J Cancer. 2006; 119: 1732-1735.

45. Li L, Li JG, Liu CY, Ding YJ. Effect of CYP1A1 and GSTM1 genetic polymorphisms on bone tumor susceptibility. Genet Mol Res. 2015; 14: 16600-16607.

46. Liu Y, He Z, Feng D, Shi G, Gao R, Wu X, Song W, Yuan W. Cytotoxic T-lymphocyte antigen-4 polymorphisms and susceptibility to osteosarcoma. DNA Cell Biol. 2011; 30: 1051-1055.

47. Liu Y, Lv B, He Z, Zhou Y, Han C, Shi G, Gao R, Wang C, Yang L, Song H, Yuan W. Lysyl oxidase polymorphisms and susceptibility to osteosarcoma. PLoS One. 2012; 7: e41610.

48. Lu H, Zhu L, Lian L, Chen M, Shi D, Wang K. Genetic variations in the PRKCG gene and osteosarcoma risk in a Chinese population: a case-control study. Tumour Biol. 2015; 36: 5241-5247.

49. Lu XF, Yang WL, Wan ZH, Li J, Bi ZG. Glutathione S-transferase polymorphisms and bone tumor risk in China. Asian Pac J Cancer Prev. 2011; 12: 3357-3360.

50. Lv H, Pei J, Liu H, Wang H, Liu J. A polymorphism site in the premiR34a coding region reduces miR34a expression and promotes osteosarcoma cell proliferation and migration. Mol Med Rep. 2014; 10: 2912-2916.

51. Ma X, Zhang Y, Sun TS, Yao JH. Role of ERCC2 and ERCC3 gene polymorphisms in the development of osteosarcoma. Genet Mol Res. 2016; 15: 10.4238/ gmr.15017302.

52. Martinelli M, Parra A, Scapoli L, De Sanctis P, Chiadini V, Hattinger C, Picci P, Zucchini C, Scotlandi K. CD99 polymorphisms significantly influence the probability to develop Ewing sarcoma in earlier age and patient disease progression. Oncotarget. 2016; 7: 77958-77967. https://doi. org/10.18632/oncotarget.12862.

53. Miao C, Liu D, Zhang F, Wang Y, Zhang Y, Yu J, Zhang Z, Liu G, Li B, Liu X, Luo C. Association of FPGS genetic polymorphisms with primary retroperitoneal liposarcoma. Sci Rep. 2015; 5: 9079.

54. Mirabello L, Berndt SI, Seratti GF, Burdett L, Yeager M, Chowdhury S, Teshome K, Uzoka A, Douglass C, Hayes RB, Hoover RN, Savage SA, National Osteosarcoma Etiology Study Group. Genetic variation at chromosome 8 q24 in osteosarcoma cases and controls. Carcinogenesis. 2010; 31: 1400-1404. 
55. Mirabello L, Yu K, Berndt SI, Burdett L, Wang Z, Chowdhury S, Teshome K, Uzoka A, Hutchinson A, Grotmol T, Douglass C, Hayes RB, Hoover RN, et al. A comprehensive candidate gene approach identifies genetic variation associated with osteosarcoma. BMC Cancer. 2011; 11: 209-2407-11-209.

56. Nakayama R, Sato $Y$, Masutani M, Ogino H, Nakatani F, Chuman H, Beppu Y, Morioka H, Yabe H, Hirose H, Sugimura H, Sakamoto H, Ohta T, et al. Association of a missense single nucleotide polymorphism, Cys1367Arg of the WRN gene, with the risk of bone and soft tissue sarcomas in Japan. Cancer Sci. 2008; 99: 333-339.

57. Naumov VA, Generozov EV, Solovyov YN, Aliev MD, Kushlinsky NE. Association of FGFR3 and MDM2 gene nucleotide polymorphisms with bone tumors. Bull Exp Biol Med. 2012; 153: 869-873.

58. Oliveira ID, Petrilli AS, Tavela MH, Zago MA, de Toledo SR. TNF-alpha, TNF-beta, IL-6, IL-10, PECAM-1 and the MPO inflammatory gene polymorphisms in osteosarcoma. J Pediatr Hematol Oncol. 2007; 29: 293-297.

59. Ozger H, Kilicoglu O, Yilmaz H, Ergen HA, Yaylim I, Zeybek U, Isbir T. Methylenetetrahydrofolate reductase C677T gene polymorphism in osteosarcoma and chondrosarcoma patients. Foli Biol (Krakow). 2008; 54: 53-57.

60. Patio-Garcia A, Sotillo-Pieiro E, Modesto C, SierrasesMaga L. Analysis of the human tumour necrosis factoralpha (TNFalpha) gene promoter polymorphisms in children with bone cancer. J Med Genet. 2000; 37: 789-792.

61. Pillay N, Plagnol V, Tarpey PS, Lobo SB, Presneau N, Szuhai K, Halai D, Berisha F, Cannon SR, Mead S, Kasperaviciute D, Palmen J, Talmud PJ, et al. A common single-nucleotide variant in $\mathrm{T}$ is strongly associated with chordoma. Nat Genet. 2012; 44: 1185-1187.

62. Qi Y, Zhao C, Li H, Zhang B, Tada K, Abe H, Tada M. Genetic variations in interleukin-6 polymorphism and the association with susceptibility and overall survival of osteosarcoma. Tumour Biol. 2016; 37: 9807-9811.

63. Qu WR, Wu J, Li R. Contribution of the GSTP1 gene polymorphism to the development of osteosarcoma in a Chinese population. Genet Mol Res. 2016; 15: 10.4238/ gmr.15038034.

64. Ru JY, Cong Y, Kang WB, Yu L, Guo T, Zhao JN. Polymorphisms in TP53 are associated with risk and survival of osteosarcoma in a Chinese population. Int J Clin Exp Pathol. 2015; 8: 3198-3203.

65. Ruza E, Sotillo E, Sierrasesumaga L, Azcona C, PatinoGarcia A. Analysis of polymorphisms of the vitamin D receptor, estrogen receptor, and collagen Ialpha1 genes and their relationship with height in children with bone cancer. J Pediatr Hematol Oncol. 2003; 25: 780-786.

66. Saito T, Krutovskikh V, Marion MJ, Ishak KG, Bennett WP, Yamasaki H. Human hemangiosarcomas have a common polymorphism but no mutations in the connexin 37 gene. Int J Cancer. 2000; 86: 67-70.
67. Salinas-Souza C, Petrilli AS, de Toledo SR. Glutathione S-transferase polymorphisms in osteosarcoma patients. Pharmacogenet Genom. 2010; 20: 507-515.

68. Savage SA, Woodson K, Walk E, Modi W, Liao J, Douglass C, Hoover RN, Chanock SJ, National Osteosarcoma Etiology Study Group. Analysis of genes critical for growth regulation identifies Insulin-like Growth Factor 2 Receptor variations with possible functional significance as risk factors for osteosarcoma. Cancer Epidemiol Biomark Prevent. 2007; 16: 1667-1674.

69. Savage SA, Burdett L, Troisi R, Douglass C, Hoover RN, Chanock SJ, National Osteosarcoma Etiology study group. Germ-line genetic variation of TP53 in osteosarcoma. Pediatr Blood Cancer. 2007; 49: 28-33.

70. Shi ZW, Wang JL, Zhao N, Guan Y, He W. Single nucleotide polymorphism of hsa-miR-124a affects risk and prognosis of osteosarcoma. Cancer Biomark. 2016; 17: 249-257.

71. Silva DS, Sawitzki FR, De Toni EC, Graebin P, Picanco JB, Abujamra AL, de Farias CB, Roesler R, Brunetto AL, Alho CS. Ewing's sarcoma: analysis of single nucleotide polymorphism in the EWS gene. Gene. 2012; 509: 263-266.

72. Tang YJ, Wang JL, Nong LG, Lan CG, Zha ZG, Liao PH. Associations of IL-27 polymorphisms and serum IL-27p28 levels with osteosarcoma risk. Medicine. 2014; 93: e56.

73. Thurow HS, Hartwig FP, Alho CS, Silva DS, Roesler R, Abujamra AL, de Farias CB, Brunetto AL, Horta BL, Dellagostin OA, Collares T, Seixas FK. Ewing Sarcoma: influence of TP53 Arg72Pro and MDM2 T309G SNPs. Mol Biol Rep. 2013; 40: 4929-4934.

74. Tian Q, Jia J, Ling S, Liu Y, Yang S, Shao Z. A causal role for circulating miR-34b in osteosarcoma. Eur J Surg Oncol. 2014; 40: 67-72.

75. Tie Z, Bai R, Zhai Z, Zhang G, Zhang H, Zhao Z, Zhou D, Liu W. Single nucleotide polymorphisms in VEGF gene are associated with an increased risk of osteosarcoma. Int J Clin Exp Pathol. 2014; 7: 8143-8149.

76. Toffoli G, Biason P, Russo A, De Mattia E, Cecchin E, Hattinger CM, Pasello M, Alberghini M, Ferrari C, Scotlandi K, Picci P, Serra M. Effect of TP53 Arg72Pro and MDM2 SNP309 polymorphisms on the risk of high-grade osteosarcoma development and survival. Clin Cancer Res. 2009; 15: 3550-3556.

77. Walsh KM, Whitehead TP, de Smith AJ, Smirnov IV, Park M, Endicott AA, Francis SS, Codd V, Samani NJ, Metayer C, Wiemels JL, ENGAGE Consortium Telomere Group. Common genetic variants associated with telomere length confer risk for neuroblastoma and other childhood cancers. Carcinogenesis. 2016; 37: 576-582.

78. Wang J, Zhou Y, Feng D, Yang H, Li F, Cao Q, Wang A, Xing F. CD86 +1057G/A polymorphism and susceptibility to Ewing's sarcoma: a case-control study. DNA Cell Biol. 2012; 31: 537-540.

79. Wang J, Nong L, Wei Y, Qin S, Zhou Y, Tang Y. Association of interleukin-12 polymorphisms and serum IL-12p40 
levels with osteosarcoma risk. DNA Cell Biol. 2013; 32: 605-610.

80. Wang K, Wang L, Feng J, Hao S, Tian K, Wu Z, Zhang L, Jia G, Wan H, Zhang J. WRN Cys1367Arg polymorphism is not associated with skull base chordoma. Biomed Rep. 2014; 2: 521-524.

81. Wang K, Zhao J, He M, Fowdur M, Jiang T, Luo S. Association of GRM4 gene polymorphisms with susceptibility and clinicopathological characteristics of osteosarcoma in Guangxi Chinese population. Tumour Biol. 2016; 37: 1105-1112.

82. Wang W, Song H, Liu J, Song B, Cao X. CD86 + 1057G/A polymorphism and susceptibility to osteosarcoma. DNA Cell Biol. 2011; 30: 925-929.

83. Wang W, Wang J, Song H, Liu J, Song B, Cao X. Cytotoxic T-lymphocyte antigen-4 $+49 \mathrm{G} / \mathrm{A}$ polymorphism is associated with increased risk of osteosarcoma. Genet Test Mol Biomark. 2011; 15: 503-506.

84. Wang Z, Wen P, Luo X, Fang X, Wang Q, Ma F, Lv J. Association of the vascular endothelial growth factor (VEGF) gene single-nucleotide polymorphisms with osteosarcoma susceptibility in a Chinese population. Tumour Biol. 2014; 35: 3605-3610.

85. Wu Y, Zhao J, He M. Correlation between TGF-beta1 gene $29 \mathrm{~T}>\mathrm{C}$ single nucleotide polymorphism and clinicopathological characteristics of osteosarcoma. Tumour Biol. 2015; 36: 5149-5156.

86. Wu Z, Wang K, Wang L, Feng J, Hao S, Tian K, Zhang L, Jia G, Wan H, Zhang J. The brachyury Gly177Asp SNP is not associated with a risk of skull base chordoma in the Chinese population. Int J Mol Sci. 2013; 14: 21258-21265.

87. Xin DJ, Shen GD, Song J. Single nucleotide polymorphisms of HER2 related to osteosarcoma susceptibility. Int J Clin Exp Pathol. 2015; 8: 9494-9499.

88. Xu H, Zhan W, Chen Z. Ras-Association Domain Family 1 Isoform A (RASSF1A) Gene Polymorphism rs1989839 is Associated with Risk and Metastatic Potential of Osteosarcoma in Young Chinese Individuals: A MultiCenter, Case-Control Study. Med Sci Monitor. 2016; 22: 4529-4535.

89. Xu S, Yang S, Sun G, Huang W, Zhang Y. Transforming growth factor-beta polymorphisms and serum level in the development of osteosarcoma. DNA Cell Biol. 2014; 33: 802-806.

90. Yang L, An Y, Wang G, Lu T, Yang S. Association between XRCC3 Thr241Met polymorphism and risk of osteosarcoma in a Chinese population. Int J Clin Exp Pathol. 2015; 8: 11670-11674.

91. Yang S, Wang C, Zhou Y, Sun G, Zhu D, Gao S. Cytotoxic T-lymphocyte antigen-4 polymorphisms and susceptibility to Ewing's sarcoma. Genet Test Mol Biomark. 2012; 16: 1236-1240.

92. Yang W, He M, Zhao J, Wang Z. Association of ITGA3 gene polymorphisms with susceptibility and clinicopathological characteristics of osteosarcoma. Med Oncol. 2014; 31 : 826-013-0826-y.

93. Zhang G, Bai R, Zhang T, Zhang H, Wen SZ, Jiang DM. Investigation of the role of VEGF gene polymorphisms in the risk of osteosarcoma. Genet Mol Res. 2015; 14: 8283-8289.

94. Zhang HF, Yan JP, Zhuang YS, Han GQ. Association between angiogenic growth factor genetic polymorphisms and the risk of osteosarcoma. Genet Mol Res. 2015; 14: 10524-10529.

95. Zhang N, Jiang Z, Ren W, Yuan L, Zhu Y. Association of polymorphisms in WWOX gene with risk and outcome of osteosarcoma in a sample of the young Chinese population. Oncotargets Ther. 2016; 9: 807-813.

96. Zhang Y, Hu X, Wang HK, Shen WW, Liao TQ, Chen P, Chu TW. Single-nucleotide polymorphisms of the PRKCG gene and osteosarcoma susceptibility. Tumour Biol. 2014; 35: 12671-12677.

97. Zhao J, Xu H, He M, Wang Z, Wu Y. Rho GTPase-activating protein 35 rs 1052667 polymorphism and osteosarcoma risk and prognosis. Biomed Res Int. 2014; 2014: 396947.

98. Zhi LQ, Ma W, Zhang H, Zeng SX, Chen B. Association of RECQL5 gene polymorphisms and osteosarcoma in a Chinese Han population. Tumour Biol. 2014; 35: 3255-3259.

99. Mei J, Huang R, Gao F, Sun H. Association between CTLA-4 polymorphisms and osteosarcoma susceptibility. Int J Clin Exp Pathol. 2016; 9: 2265-2270.

100. Mocellin S, Rossi CR. The melanoma molecular map project. Melanoma Res. 2008; 18: 163-165.

101. Zhang B, Beeghly-Fadiel A, Long J, Zheng W. Genetic variants associated with breast-cancer risk: comprehensive research synopsis, meta-analysis, and epidemiological evidence. The Lancet.Oncology. 2011; 12: 477-488.

102. Theodoratou E, Montazeri Z, Hawken S, Allum GC, Gong J, Tait V, Kirac I, Tazari M, Farrington SM, Demarsh A, Zgaga L, Landry D, Benson HE, et al. Systematic metaanalyses and field synopsis of genetic association studies in colorectal cancer. J Nat Cancer Instit. 2012; 104: 1433-1457.

103. Mocellin S, Verdi D, Pooley KA, Nitti D. Genetic variation and gastric cancer risk: a field synopsis and meta-analysis. Gut. 2015; 64: 1209-1219.

104. Brohl AS, Patidar R, Turner CE, Wen X, Song YK, Wei JS, Calzone KA, Khan J. Frequent inactivating germline mutations in DNA repair genes in patients with Ewing sarcoma. Genet Med. 2017; 19: 955-958.

105. Sholl LM, Barletta JA, Hornick JL. Radiation-associated neoplasia: clinical, pathological and genomic correlates. Histopathology. 2017; 70: 70-80.

106. Kadouri L, Sagi M, Goldberg Y, Lerer I, Hamburger T, Peretz T. Genetic predisposition to radiation induced sarcoma: possible role for BRCA and p53 mutations. Breast Cancer Res Treat. 2013; 140: 207-211. 
107. Berwick M, Song Y, Jordan R, Brady MS, Orlow I. Mutagen sensitivity as an indicator of soft tissue sarcoma risk. Environ Mol Mutagen. 2001; 38: 223-226.

108. Stewart E, Goshorn R, Bradley C, Griffiths LM, Benavente C, Twarog NR, Miller GM, Caufield W, Freeman BB 3rd, Bahrami A, Pappo A, Wu J, Loh A, et al. Targeting the DNA repair pathway in Ewing sarcoma. Cell Rep. 2014; 9: 829-841.

109. Little J, Higgins J, Bray M, Ioannidis J, Khoury M, Manolio T, Smeeth L, Sterne J. The HuGENet ${ }^{\mathrm{TM}}$ HuGE review handbook, version 1.0. Ottawa, Ontario, Canada: HuGENet Canada Coordinating Centre. 2006.

110. Stang A. Critical evaluation of the Newcastle-Ottawa scale for the assessment of the quality of nonrandomized studies in meta-analyses. Eur J Epidemiol. 2010; 25: 603-605.

111. Ioannidis JP, Boffetta P, Little J, O'Brien TR, Uitterlinden AG, Vineis P, Balding DJ, Chokkalingam A, Dolan SM, Flanders WD, Higgins JP, McCarthy MI, McDermott $\mathrm{DH}$, et al. Assessment of cumulative evidence on genetic associations: interim guidelines. Int J Epidemiol. 2008; 37: 120-132.

112. Wacholder S, Chanock S, Garcia-Closas M, El Ghormli L, Rothman N. Assessing the probability that a positive report is false: an approach for molecular epidemiology studies. Journal of the National Cancer Institute. 2004; 96: 434-442.

113. Stacey SN, Sulem P, Masson G, Gudjonsson SA, Thorleifsson G, Jakobsdottir M, Sigurdsson A, Gudbjartsson DF, Sigurgeirsson B, Benediktsdottir KR, Thorisdottir K, Ragnarsson R, Scherer D, et al. New common variants affecting susceptibility to basal cell carcinoma. Nat Genet. 2009; 41: 909-914.

114. GTEx Consortium. Human genomics. The GenotypeTissue Expression (GTEx) pilot analysis: multitissue gene regulation in humans. Science. 2015; 348: 648-660.

115. Szklarczyk D, Franceschini A, Wyder S, Forslund K, Heller D, Huerta-Cepas J, Simonovic M, Roth A, Santos A, Tsafou KP, Kuhn M, Bork P, Jensen LJ, et al. STRING v10: protein-protein interaction networks, integrated over the tree of life. Nucleic Acids Res. 2015; 43: D447-52.

116. Kuleshov MV, Jones MR, Rouillard AD, Fernandez NF, Duan Q, Wang Z, Koplev S, Jenkins SL, Jagodnik KM, Lachmann A, McDermott MG, Monteiro CD, Gundersen GW, et al. Enrichr: a comprehensive gene set enrichment analysis web server 2016 update. Nucleic Acids Res. 2016; 44: W90-7. 